STONE CENTER ON SOCIO-ECONOMIC INEQUALITY

WORKING PAPER SERIES

No. 37

Chasing the Tail:

A Generalized Pareto Distribution Approach to Estimating Wealth Inequality

Arthur B. Kennickell

May 2021

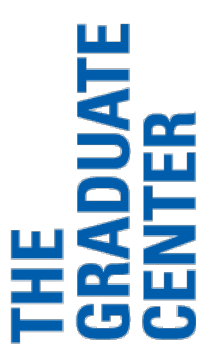


Preliminary

\title{
Chasing the Tail: A Generalized Pareto Distribution Approach to Estimating Wealth Inequality
}

\author{
Arthur B. Kennickell \\ Stone Center, CUNY Graduate Center \\ Arthur.Kennickell@gmail.com
}

May 10, 2021

\begin{abstract}
Since the work reported in Vermeulen [2018], a literature has developed on using the simple Pareto distribution along with "rich list" information to make improved estimates of the upper tail of the wealth distribution measured in surveys. Because the construction of such external data is typically opaque and subject to potentially serious measurement error, it may be best not to depend exclusively on this approach. This paper develops an alternative approach, using the generalized Pareto distribution (GPD), of which the simple Pareto is a subset, extending an estimation strategy developed by Castillo and Hadi [1997]. The greater flexibility of the GPD allows the possibility of modeling the tail of the wealth distribution, using a larger set of data for support than is typically the case with the simple Pareto. Moreover, the elaboration of the estimation method presented here allows explicitly for the possibility that the extreme of the observed upper tail is measured with error or that it is not captured at all. The approach also allows the incorporation of external data on total wealth as a constraint on the estimation. For the applications considered here using Austrian and U.S. micro data, the model relies on an estimate of total household wealth from national accounts, rather than rich-list information. The results suggest that where sufficiently comparable and reliable estimates of aggregate wealth are available, this approach can provide a useful way of mitigating problems in comparing distributional estimates across surveys that differ meaningfully in their effective coverage of the upper tail of the wealth distribution. The approach may be particularly useful in the construction of distributional national accounts.
\end{abstract}

The author is grateful to the Stone Center at the CUNY Graduate Center for computer and organizational support. The author is also grateful for helpful comments from Pirmin Fessler, Bertrand Garbinti, Martin Schürz and Philip Vermeulen. The author alone is responsible for any errors or oversights. 
This paper was primarily motivated by the need to address differences in the measurement of the upper tail of the wealth distribution across surveys in a flexible way that allows explicitly for imperfect measurement. For example, the Household Finance and Consumption Survey (HFCS) consists of 20 country-level surveys, most of which attempt to oversample the wealthiest part of the population in some way. ${ }^{1}$ However, owing largely to great differences across countries in the types of information available to do so, they meet with widely varying degrees of success. ${ }^{2}$ Nonrandom nonresponse among wealthy households may further diminish the ability of a sample to capture the upper tail (see Vermeulen, 2018). Although such differences have minimal importance for estimating characteristics and behavior of the great majority of the population, they do have important implications for the ability to estimate a variety of measures of inequality (see Kennickell, 2019) or to make use of the data in developing distributional national accounts (see Cantarella et al. [2021]). Because the upper tail of the wealth distribution is typically very skewed, relatively small differences across surveys in their effective coverage of the upper tail can result in quite different levels of accuracy and estimated levels of precision.

One way to address this problem is to use a fitted distribution to project the extreme tail. The simple Pareto distribution is often used to describe the upper tail of such skewed economic distributions as those of income or wealth; for example, Atkinson [1975] made pioneering use of the simple Pareto distribution to model wealth data from estate-tax records. It is relatively straightforward to fit this one-parameter distribution and it has had application in extrapolating the upper tail of distributions for which external information is available, such as lists of the wealth of the wealthiest people in a country, as discussed further below. Often, in this context the Pareto is fit on data above a point in the top of the wealth distribution. In such cases, the potential for broader, smooth integration with the remainder of the distribution is often limited.

This paper develops an alternative approach, using the Generalized Pareto Distribution (GPD), of which the simple Pareto is a special case. ${ }^{3}$ The GPD is widely used to model extreme events, from floods to financial market behavior. ${ }^{4}$ By virtue of its more flexible functional form, the GPD has greater potential to fit the data within the bulk of a sample. As shown below, by extending the estimation approach of Castillo and Hadi [1997], the GPD has the potential to cope with data which are subject to measurement error, are incorrectly covered by the sample, or are not observed at all as a result of differential nonresponse, errors or omissions in the survey sample, or random variation in sampling. This approach also offers a straightforward means of incorporating external information on the aggregate amount of wealth as a constraint on the estimation, a feature particularly relevant for the construction of distributional national accounts.

The first section of this paper reviews a small selection of the growing number of papers that have used fitted distributions to address the defects in measurement of the upper tail of the wealth distribution in surveys. The second section presents the technical methodology developed here for applying the GPD to such problems. The third section uses data from the 2017 Austrian HFCS and the 2016 U.S. Survey of

\footnotetext{
${ }^{1}$ See European Central Bank [2020a and 2020b].

${ }^{2}$ Ideally, one would want to design an over sample of wealthy households, based on household-specific wealth information (such as from wealth tax data) or other data highly correlated with wealth (such as types of capital income), and use that frame information to adjust for nonresponse.

${ }^{3}$ A GPD with shape $\mathrm{k}<0$ and location $\lambda^{0}=-\sigma / k$, it is equivalent to a Pareto distribution with $x_{m}=-\sigma / k$ and shape $\alpha=-1 / k$.

${ }^{4}$ The GPD should not be confused with a generalized Pareto curve (Blanchet et al., 2018) which treats the shape coefficient in the simple Pareto distribution as potentially variable across areas of a distribution.
} 
Consumer Finances (SCF) to demonstrate the possibilities for applying the approach. The final section concludes and points to further research.

\section{Related work in wealth distribution modeling}

The work reported in Vermeulen [2018] opened a line of research aimed at adjusting the upper tail of the wealth distribution measured in surveys, using eternal data derived from country-specific lists of very wealthy people. ${ }^{5}$ The paper shows, convincingly, that nonresponse bias together with an inadequate sampling rate in the highly skewed tail of the wealth distribution can lead to a very unrepresentative picture of the overall wealth distribution. Adapting the simple Pareto distribution to differentially sampled survey data, the paper characterizes the effects of supplementing the survey observations with cases from a "rich list". For surveys without a strong means of over sampling wealthy households, the inclusion of the supplemental observations substantially alters the Pareto estimates and indicators of wealth concentration. It has a smaller effect on surveys that are able to oversample in this way.

The change implied by this addition almost certainly represents some improvement in addressing the upper tail, given that it is obvious that such very wealthy observations are not present at all in most surveys. Nonetheless, there is no guarantee that the implied aggregate wealth would correspond to a conceptually comparable estimate from national accounts, though at the time (and still even now) such estimates were not yet determined for many countries. While use of rich lists for this purpose may be a reasonable expedient, it may not be the best long-term solution. Rich lists are generally opaque in their construction, relying on publicly available data, informal information and sometimes cooperation from the parties included. Moreover, it is often difficult to identify the wealth of individual wealthy households, as opposed to extended families or family foundations; wealth may be over- or under-stated; and some households may act in ways that disguise or even exaggerate their potential for inclusion in such a list. For example, the U.S. Survey of Consumer Finances (SCF) by design specifically excludes members of the Forbes list of the 400 wealthiest people in the U.S., but it routinely finds households wealthier than the least wealthy people on that list. ${ }^{6}$

For purposes of comparison with estimates given in the present paper, it is useful to note that the Vermeulen paper provides estimates of the wealth share for the wealthiest one percent of household for 2010 based on the Austrian HFCS and the SCF. For Austria, the share rises from the 23 percent figure computed from the unadjusted data to 31-32 percent; the comparable figures for the SCF are 34 percent and a range of $31-37$ percent.

Chakraborty et al. [2019] focuses most on understanding key differences between survey and national account measures of wealth and developing an approach for bridging the gap. The paper recognized a long line of research aimed at understanding the conceptual differences between wealth measured in surveys and that measured in national accounts. This work has so far been conducted most intensively in the U.S., using data from the SCF and the Financial Accounts of the U.S., beginning with Avery et al. [1988] and continuing through, among others, Antoniewicz [2000], Henriques and Hsu [2014], Detling et al. [2015] and, most recently, Batty et al. [2019]; interest in distributional national accounts has accelerated work in this area in Europe, as exemplified by Kavonius and Törmälehto [2010] and Cantarella et al. [2021]. Such work will be essential if distributional national accounts are to be widely accepted. It is clear from all this work that careful alignment of the survey and aggregate concepts can

\footnotetext{
${ }^{5}$ The associated working paper dates from 2014.

${ }^{6}$ In addition, Raub et al. [2010], using an exact match of Forbes and estate tax data, provide a detailed discussion of valuation differences, and Kennickell [1999] based on an exact match of Forbes data and income from administrative data to question the Forbes rankings.
} 
lead to a substantial improvement in the match of asset levels. But such adjustments alone are not necessarily sufficient to align the totals. Following the methodology of Vermeulen [2018], Chakraborty et al. use an external rich list to make a Pareto extrapolation of the HFCS wealth distribution for several countries, with similar results. An important contribution of the paper. is its focus on the effects of the Pareto adjustments on sub-categories of net worth. Even if net worth can be fully aligned conceptually and empirically in a survey and in aggregate data through a distributional extrapolation, addressing remaining differences at the level of wealth components remains an important question, one that is also left open by the current paper.

Bricker et al. [2019] also apply a Pareto methodology to estimate measures of wealth concentration for years corresponding to various years of the SCF. As noted in more detail later in this paper, the SCF has an unusually high rate of coverage of the top of the wealth distribution, excluding the Forbes group. For the current paper, the Bricker et al. paper is an important point of reference, since it uses the non-public version of the SCF data, which unlike the public version, includes all the observations (including those with wealth greater than the Forbes minimum wealth) and variables that have not been altered for purposes of disclosure limitation. According to the authors' preferred model, which blends the observations in the Forbes list and the survey observations in the range of overlap, the share of wealth held by the wealthiest one percent in 2016 was 40.3 percent, with a 95 percent confidence interval of $(38.4,42.2)$. In this model, the minimum wealth from which the Pareto distribution was fit was $\$ 8.8$ million, or about the $98.7^{\text {th }}$ percentile of net worth, as observed in the public version of the data.

\section{Methodology}

\subsection{Background on the GPD}

Much of the original technical development of the GPD was done by Picklands [1965]. Hosking and Wallis [1987] provide a broad description of the properties of the GPD. The cumulative distribution function for the GPD is defined as

$$
F(\mathrm{~V})=F\left(\lambda-\lambda^{0} \mid \lambda>\lambda^{0}>0\right)=1-\left(1-\frac{k\left(\lambda-\lambda^{0}\right)}{\sigma}\right)^{\frac{1}{k}},
$$

where $k$ is taken to be the shape parameter, $\sigma$ the scale parameter and $\lambda^{0}$ the location parameter. ${ }^{7} \mathrm{~V}$ is commonly referred to as the vector of "exceedances" of $\lambda$ relative to $\lambda^{0}$, the lowest value for which the distribution is taken to apply. As shown in figure 1, higher values of $\sigma$ and higher absolute values of $k$ both make for a fatter right tail, but their nonlinear relationship allows them to trade off in fitting data. ${ }^{8}$ Note that even if two GPDs have the same mean, they may have very different shapes, as in the case of the distributions plotted in figure 2, and thus differences in implied measures of concentration.

A property of the generalized Pareto distribution that turns out to be especially useful for the purposes of this paper is that when $k>-1$, the mean of the distribution of the GPD is given by

\footnotetext{
${ }^{7}$ When $k=0$, the distribution reduces to the exponential distribution. This case is not relevant in this paper and it will be ignored in what follows. A GPD with shape $\mathrm{k}<0$ and location $\lambda^{0}=-\sigma / k$, is equivalent to a Pareto distribution with cumulative distribution given by $P(x)=1-\left(\frac{x_{m}}{x}\right)^{\alpha}$ with $x_{m}=-\sigma / k$ and shape $\alpha=-1 / k$.

${ }^{8}$ For simplicity, $\lambda^{0}$ is taken to be zero in the figures 1 and 2 . The figure focuses on the top 1 percent of the distribution in each case, in order to highlight the differences graphically. In figure 1, the mean of each distribution is as follows: ; $\mathrm{k}=-0.5$, sigma $=1000$ : mean $=2000 ; \mathrm{k}=-0.75$, sigma $=1000$ : mean $=4000 ; \mathrm{k}=-0.95$, sigma $=1000$ : mean $=20000 ; \mathrm{k}=-0.75$, sigma $=500$ : mean $=2000 ; \mathrm{k}=-0.75$, sigma $=2000,:$ mean $=8000$.
} 


$$
E\left[\lambda-\lambda^{0} \mid \lambda>\lambda^{0}>0\right] \equiv \mu_{\lambda^{0}}=\frac{\sigma}{1+k}, \text { if } k>-1 .
$$

In addition, for any $\lambda^{+}>\lambda^{0}$ the distribution of $\lambda-\lambda^{+}$for $\lambda>\lambda^{+}$remains GPD with the same shape parameter $k$ and with the scale parameter given by $\sigma-k\left(\lambda^{+}-\lambda^{0}\right)$. By substitution into (2) we have

$$
\begin{gathered}
E\left[\lambda-\lambda^{+} \mid \lambda>\lambda^{+}>\lambda^{0}\right] \equiv \mu_{\lambda^{+}}=\frac{\sigma-k\left(\lambda^{+}-\lambda^{0}\right)}{1+k}=\alpha+\beta \lambda^{+}, \text {where } \\
\alpha=\frac{\sigma+k \lambda^{0}}{1+k} \text { and } \beta=-\frac{k}{1+k} .
\end{gathered}
$$

Figure 1: GPD for various parameter values

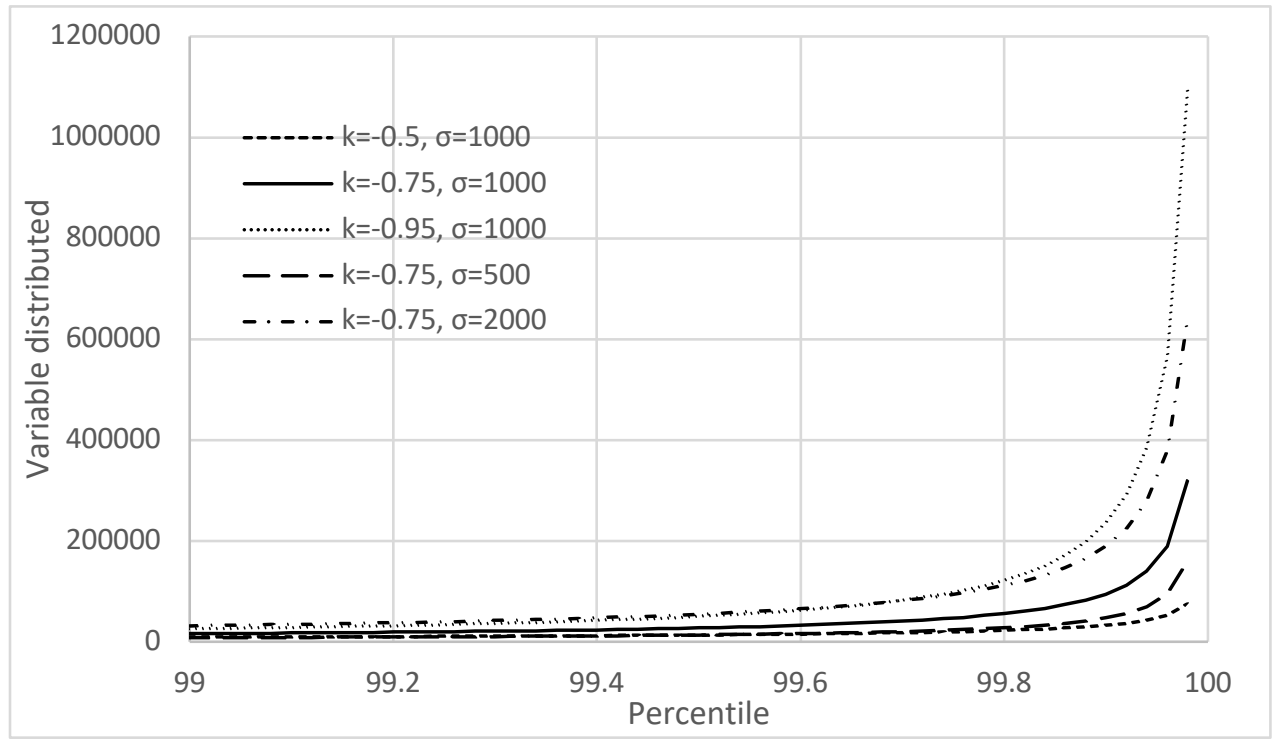

Figure 2: Selected GPDs with the same mean

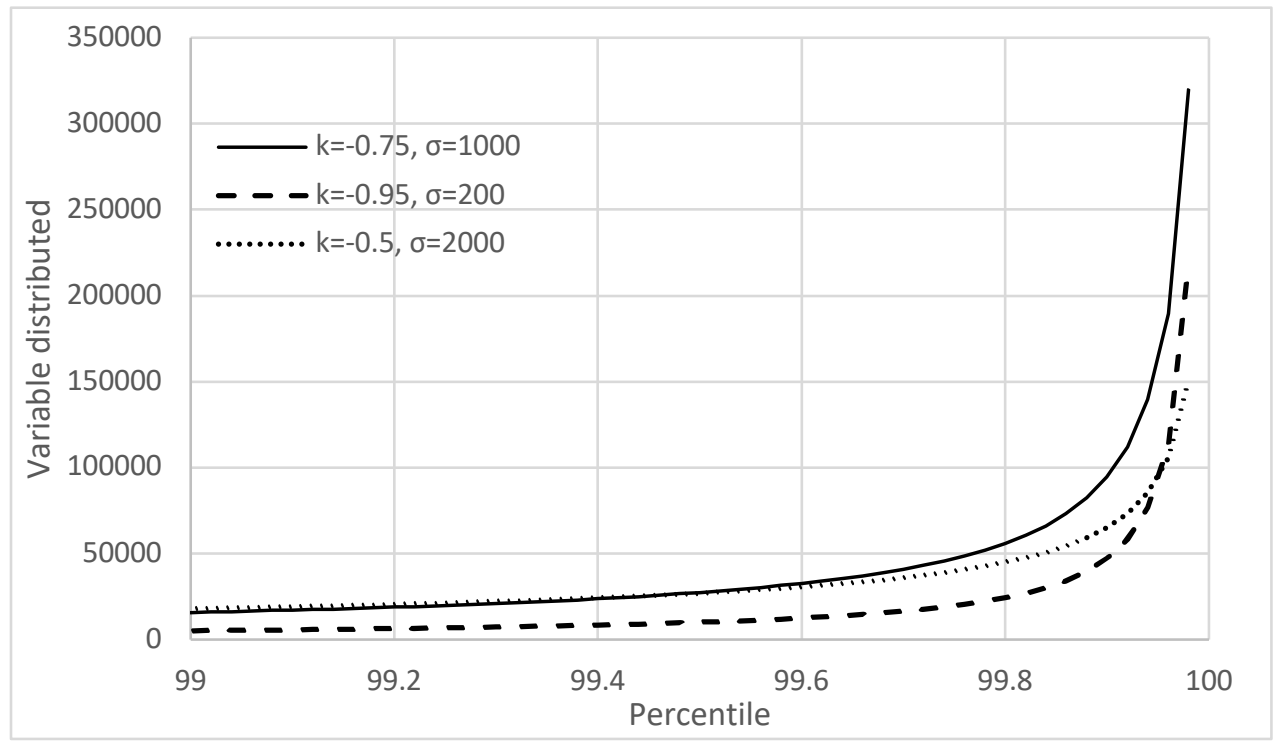


Thus, there is a linear relationship between the mean of the exceedances of all values greater than any arbitrary $\lambda^{+}>\lambda^{0}$, where the exceedances are defined relative to $\lambda^{+}$, and the value of $\lambda^{+}$. As shown later in the empirical part of this paper, this linear relationship provides a useful diagnostic tool for exploring empirical data for the potential for applying the GPD. ${ }^{9}$

Estimation of the parameters of a GPD may be performed by maximum likelihood, method of moments, or another goodness of fit criterion, using the observed values of $\lambda$. However, as Hosking and Wallis [1987] note, in some circumstances, maximum likelihood and method of moments estimates may not exist or may be inconsistent with observed data or otherwise unreliable. In addition, irregularities or mismeasurements in data may make estimation difficult or yield implausible results. For purposes of the current paper, the important such issues concern the extreme upper tail and the extent to which it is either not fully observed or inadequately measured in some other way. The approach developed by Castillo and Hadi [1997] and placed in a more general framework by Brazauskas and Kleefeld [2009] offers are more flexible and robust means of fitting a GPD, which is elaborated here to deal with problems of this type.

\subsection{Castillo and Hadi approach}

As adapted here for survey data, the Castillo-Hadi $(\mathrm{CH})$ approach involves using pairs of empirical percentile points and their corresponding order statistics to solve for the GPD parameters implied by the interval and repeating that process over a large number of other pairs to obtain a set of possible estimates from which the final estimates are selected. ${ }^{10}$ In principle, the pairs may be chosen from any part of the range of the distribution. This aspect of flexibility turns out to be especially useful for fitting the GPD to empirical distributions that may have relatively unreliable information in some part of the range. As will be shown below, the approach also lends itself to a straightforward adaptation for dealing with the possibility that a certain part of a distribution, for example the wealthiest $1 / 2$ percent, is not observed at all.

The method of estimation follows from the substitution $\delta=\sigma / k$ (for $k \neq 0)$ in (1) to yield

$$
F\left(\lambda-\lambda^{0}\right)=1-\left(1-\frac{\left(\lambda-\lambda^{0}\right)}{\delta}\right)^{\frac{1}{k}} .
$$

Given the observed values $\Lambda=\left\{\lambda_{1}, \ldots, \lambda_{n}\right\}$, and the corresponding empirical percentile values of the empirical distribution of $\Lambda, F(\Lambda) \equiv P=\left\{p_{1}, \ldots, p_{n}\right\}$, it is then straightforward for a given $i$ to solve for $k$ and $\sigma$ in terms of each other as follows:

$$
\begin{gathered}
k=\ln \left(1-\left(\lambda_{i}-\lambda_{0}\right) / \delta\right) / \ln \left(1-p_{i}\right) \\
\delta=\left(\lambda_{i}-\lambda_{0}\right) /\left[1-\left(1-p_{i}\right)^{k}\right] .
\end{gathered}
$$

\footnotetext{
${ }^{9}$ This relationship also provides a potential means of estimating $k$ and $\sigma$, as discussed in Langousis et al. (2016). However, simulation experiments conducted as a part of this research reported in this paper indicate that the method tends to under-estimate these parameters noticeably, even in samples of several thousand simulated observations. ${ }^{10}$ Castillo and Hadi defined their approach in terms of order statistics and a corresponding percentile definition. This is straightforward to adapt to the context of values taken from a survey with variation in weights. For the $i^{\text {th }}$ order statistic $\mathrm{V}_{\mathrm{i}}$, Castillo and Hadi define the empirical percentile $p_{i}=\frac{i-\gamma}{n+\beta}$, where observations are arranged in ascending order from 1 to $\mathrm{n}$. In their application they find best results with $\gamma=0$ and $\beta=1$. In the implementation presented here, $p_{i}$ is taken to be the weighted percentile of the empirical distribution of $\Lambda$, corresponding to $\lambda_{i}$.
} 
Taking the ratio of (6) for $i$ and $j$ yields equation (8) in one unknown, which is straightforward to solve for $\delta$ by search.

$$
\frac{\ln \left(1-\left(\lambda_{i}-\lambda_{0}\right) /_{\delta}\right)}{\ln \left(1-\left(\lambda_{j}-\lambda_{0}\right) / \delta\right)}=\frac{\ln \left(1-p_{j}\right)}{\ln \left(1-p_{i}\right)}
$$

$\mathrm{CH}$ develop an explicit bound on the value of $\delta$, thus simplifying the search for the estimate. For $\lambda_{\mathrm{i}}<\lambda_{\mathrm{j}}$ the bound is defined in terms of the two following values:

$$
\begin{gathered}
\delta_{b}=\frac{\left(\lambda_{i}-\lambda_{0}\right)\left(\lambda_{j}-\lambda_{0}\right)\left(\ln \left(1-p_{j}\right)-\ln \left(1-p_{i}\right)\right)}{\left(\lambda_{i}-\lambda_{0}\right) \ln \left(1-p_{j}\right)-\left(\lambda_{j}-\lambda_{0}\right) \ln \left(1-p_{j}\right)} \\
r_{i}=\frac{\left(\lambda_{j}-\lambda_{0}\right) \ln \left(1-p_{j}\right)}{\ln \left(1-p_{j}\right)} ;
\end{gathered}
$$

if $\lambda_{i}<r_{i}$ then $\delta \in\left(\delta_{b}, 0\right)$, or if $\lambda_{i}>r_{i}$ then $\delta \in\left(\lambda, \delta_{b}\right)$. Given an estimate of $\delta$, one may then use the data points to compute the estimates of $k$ and $\sigma .{ }^{11}$ The process is repeated over other pairs of values of $\Lambda$ and the corresponding values of $\mathrm{P}{ }^{12}$

\subsection{Selecting a set of parameter estimates}

The estimation process described above yields a potentially large set of candidate parameter estimates. Except in the case where the data are exactly GPD, estimates of $k$ and $\sigma$ may differ across sets of pairs. Thus, some method is needed to select the most appropriately descriptive values. $\mathrm{CH}$ argue for selecting the median of the estimates of $k$ and $\sigma$. Where the data values supporting the estimation are selfweighting, as they are in the problem those authors address, it would be straightforward, if cumbersome with a large set of values, to compute estimates for every possible combination of values of $(\lambda, p)$. For the more general problem of working with data that are not self-weighting, such as disproportionately sampled survey data, the simple median of the estimates does not have a straightforward interpretation. Moreover, in any case there is no guarantee that the separately determined values of $k$ and $\sigma$ would correspond to the same value of $(\lambda, p)$.

The approach taken here is to apply a modified version of the Anderson-Darling right-tail-weighted distance (RTAD) measure (see Chernobai et al. [2005] and Hoffmann and Börner [2020]) to select a candidate pair of parameters, and then apply of an adaptation of the Cramer von Mises (CVM) test (see Choulakian and Stephens [2001]) to assess the plausibility that the observed data may be described by a

\footnotetext{
${ }^{11}$ Note that in principle, on might construct estimates of $k$ and $\sigma$ from $\delta$, using the data from either end of the interval used to construct the estimate of $\delta$. Extensive testing for this paper indicates that the choice of end point to use makes little difference. In this paper, only estimates constructed from the lower percentile and corresponding order statistics are reported.

Instead of solving for an estimate of $\delta$, one could, alternatively, solve for the value of $k$ that satisfies the following equality: $\frac{v_{i}}{v_{j}}=\frac{\left[1-\left(1-p_{i}\right)^{k}\right]}{\left[1-\left(1-p_{j}\right)^{k}\right]}$. The advantage of solving first for $\delta$ is that there is an explicit bound on its value, as noted in the text.

${ }^{12}$ As this model is implemented here, 50 equally spaced points are determined across the interval over which the estimation is performed, and 100 points randomly distributed around those points are paired for estimation with other randomly chosen points. This approach to stratifying the support of the estimation ensures that a wide set of intervals is considered. The model generates approximately 5,100 estimates of the parameters at this stage.
} 
GPD with these parameter estimates. The RTAD measure as implemented here is given in (11). In its basic form this measure computes the sum of the squared weighted difference between the cumulative probability of the distributions implied by the set of parameters given each value of the exceedances $\lambda-$ $\lambda^{0}$, and the empirical cumulative probabilities for the same values of the exceedances, where the weight is the inverse of the predicted probability. Thus, the denominator serves to differentially weight the fit of the upper tail of the distribution. Given the interest here in fitting the upper tail, this is a preferred alternative to other measures, such as the Kolmogorov-Smirnov, Cramer von Mises (CVM) or the usual Anderson-Darling (weighting both tails) measures. To avoid extreme weighting, the value of $\hat{p}$ in the denominator is capped at a value of 0.99 . Finally, to adapt the measure for differentially weighted survey data, it is necessary to incorporate the weight $W(\lambda)$ corresponding to each sample value $\lambda$. The candidate joint estimate of $(\hat{k}, \hat{\sigma})$ is the one yielding the minimum value of $(11)$.

$$
A D_{-} R T\left(\lambda^{0}, \hat{k}, \hat{\sigma}\right)=\frac{\sum_{\lambda>\lambda^{0}} W(\lambda)\left\{\frac{\hat{p}\left(\lambda-\lambda^{0} \mid \hat{k}, \hat{\sigma}\right)-\mathrm{p}\left(\lambda-\lambda^{0}\right)}{1-\min \left(0.99, \hat{p}\left(\lambda-\lambda^{0} \mid \hat{k}, \hat{\sigma}\right)\right)}\right\}^{2}}{\sum_{\lambda>\lambda^{0}} W(\lambda)}
$$

Despite being a minimum of the RTAD criterion, the resulting estimates may not provide a plausible description of the data as GPD. The CVM test offers one way to assess this possibility. The test shown in (12) is a modification of the CVM test given by Choulakian and Stephens, which they defined in terms of order statistics. The core of the test is the same squared difference in the RTAD measure, but in this case not probability weighted. As with the RTAD measure, it is necessary here to account for the weight variation in differentially sampled data. In addition, the CVM test statistic, requires to grossing up this measure by an appropriate measure of the sample size, taken here to be the effective sample size (ESS) (see Kish [1965]). Choulakian and Stephens provide a table of test statistics for the plausibility assessment specific to the value of $\hat{k}$ (denoted here by $\operatorname{TCVM}(\hat{k})$, and they argue for using a critical pvalue of 0.1 .

$$
\begin{gathered}
C V M\left(\lambda^{0}, \hat{k}, \hat{\sigma}\right)=\sum_{\lambda>\lambda^{*}} \varpi(\lambda)\left[\hat{p}\left(\lambda-\lambda^{0} \mid \hat{k}, \hat{\sigma}\right)-\mathrm{p}\left(\lambda-\lambda^{0}\right)\right]^{2}+\frac{1}{12 \operatorname{ESS}\left(\lambda^{0}\right)} \\
\varpi(\lambda)=\frac{W(\lambda)}{\sum_{\mathrm{L}>\lambda^{0}} W(L)} \frac{\left(\sum_{L>\lambda^{0}} W(L)\right)^{2}}{\sum_{L>\lambda^{0}} W(L)^{2}}=\frac{W(\lambda)}{\sum_{\mathrm{L}>\lambda^{0}} W(L)} \operatorname{ESS}\left(\lambda^{0}\right) .
\end{gathered}
$$

\subsection{Determining the location parameter}

The exposition to this point implicitly assumes that the value of $\lambda^{0}$ has been determined by some means external to the estimation. Many distributions, including the wealth distribution that is the main focus of this paper, exhibit patterns that appear possibly GPD at the top of the distribution, but not obviously so everywhere below that. In principle, estimates incorporating the latter such points should not pass the CVM test above. Rather than use the imperfect mechanism of inspecting the data for a "reasonable" break point, here a more principled approach is used.

The relationship expressed in equation (3) offers a possible key to the selection of the region of data for modeling. As noted, if the data may be considered GPD for a given value of the location parameter, then the data for higher values of the location parameter should also be GPD with the same value of $k$ and a modified value of $\sigma$. According to this argument, for the sake of estimation efficiency the lowest value of $\lambda$ for which the resulting data are consistent with GPD should be used as $\lambda^{0}$, which is denoted here as $\lambda^{0 *}$. 
The following procedure involving the CVM test described above is one way of making this determination. As given above, compute $\left(\hat{k}_{i}, \hat{\sigma}_{i} \mid \lambda_{i}\right)$ for values of $\lambda_{i}$ greater than some $\lambda^{\text {min }}$, up to the maximum value that allows identification of the parameters $\left(\lambda^{\max }\right)$, calculate $C V M\left(\hat{k}_{i}, \hat{\sigma}_{i}, \lambda_{i}\right)$, and compare that value to the CVM test statistic $\operatorname{TCVM}\left(\hat{k}_{i}\right)$. In accord with the argument above, the lowest value of $\lambda$ that passes this test should be used as the final value of $\lambda^{0 *}$.

However, contrary to the theoretical expectation that higher values of the location parameter should also imply that the data are GPD with the same shape parameter, this may not be the case in practice. Indeed, it is clearly not so in the case of one set of data considered in next section of this paper. If values of $\operatorname{CVM}\left(\hat{k}_{i}, \hat{\sigma}_{i}, \lambda_{i}\right)$ for $\lambda_{i}>\lambda^{0 *}$ are locally noisy, loosely speaking, then it may be more efficient to smooth across the estimated parameters in the following way. As shown in (14), for $\lambda \epsilon\left(\lambda^{0 *}, \lambda^{\max }\right)$ for which the implied parameters pass the CVM test, compute the weighted average of $\hat{k}$, where the weight is the effective sample size associated with each set of estimates $(\hat{k}, \hat{\sigma})$.

$$
\hat{k}_{\text {mean }}=\frac{\sum_{\lambda \geq \lambda^{0 *}}^{\lambda_{\max }} \operatorname{ESS}(\lambda) W(\lambda)\left(\operatorname{CVM}\left(\lambda, \hat{k}_{\lambda}, \hat{\sigma}_{\lambda}\right) \leq \operatorname{TCVM}\left(\hat{k}_{\lambda}\right)\right) \hat{k}_{\lambda}}{\sum_{\lambda \geq \lambda^{0 *}}^{\lambda^{\max }} \operatorname{ESS}(\lambda) W(\lambda)\left(\operatorname{CVM}\left(\lambda, \hat{k}_{\lambda}, \hat{\sigma}_{\lambda}\right) \leq \operatorname{TCVM}\left(\hat{k}_{\lambda}\right)\right)} .
$$

Given this estimate of the shape parameter, several additional steps are needed to determine $\hat{\sigma}$ and the final selection of $\lambda^{0}$. Because $\hat{k}$ and $\hat{\sigma}$ are closely related and $\hat{\sigma}$ tends to increase with $\lambda$, a corresponding average for $\hat{\sigma}$ would not give a meaningfully reliable estimate. Instead, the approach taken here is to compute the mean of $\delta$ (defined earlier as $\sigma / k$ ), where the value of $\sigma$ for each value of $\lambda$ is "standardized" at a common value $\lambda^{s 13}$, and use the resulting mean together with $\hat{k}_{\text {mean }}$ to determine $\hat{\sigma}_{\text {mean }}$ as follows:

$$
\begin{gathered}
\sigma\left(\lambda, \lambda^{s}\right) \equiv \hat{\sigma}_{\lambda}-\hat{k}_{\lambda}\left(\lambda^{s}-\lambda\right) \\
\hat{\delta}_{\text {mean }}=\frac{\sum_{\lambda \geq \lambda^{*}}^{\lambda^{\max }} \operatorname{ESS}(\lambda) W(\lambda)\left(\operatorname{CVM}\left(\lambda, \hat{k}_{\lambda}, \hat{\sigma}_{\lambda}\right) \leq \operatorname{TCVM}\left(\hat{k}_{\lambda}\right)\right)\left(\sigma\left(\lambda, \lambda^{s}\right) / \hat{k}_{\lambda}\right)}{\sum_{\lambda \geq \lambda^{*}}^{\lambda_{\max }} \operatorname{ESS}(\lambda) W(\lambda)\left(\operatorname{CVM}\left(\lambda, \hat{k}_{\lambda}, \hat{\sigma}_{\lambda}\right) \leq \operatorname{TCVM}\left(\hat{k}_{\lambda}\right)\right)}
\end{gathered}
$$

Define the standard deviations $\hat{k}_{\text {std }}$ and $\hat{\delta}_{\text {std }}$ analogously to (14) and for each value of $\lambda$ for which $\operatorname{CVM}\left(\lambda, \hat{k}_{\lambda}, \hat{\sigma}_{\lambda}\right) \leq \operatorname{TCVM}\left(\hat{k}_{\lambda}\right)$ calculate the normalized value of $\hat{k}_{\lambda}$ and $\hat{\delta}_{\lambda}$ as

$$
\begin{aligned}
& \hat{k}_{\lambda}^{\text {norm }}=\left(\hat{k}_{\lambda}-\hat{k}_{\text {mean }}\right) / \hat{k}_{s t d} \text { and } \\
& \hat{\delta}_{\lambda}^{\text {norm }}=\left(\hat{\delta}_{\lambda}-\hat{\delta}_{\text {mean }}\right) / \hat{\delta}_{\text {std }} . \\
& \lambda^{0 *}=\operatorname{ARGMIN} \operatorname{IICVM}_{\lambda\left(\lambda, \hat{k}_{\lambda}, \hat{\sigma}_{\lambda}\right) \leq \operatorname{TCVM}\left(\hat{k}_{\lambda}\right)}\left(\hat{k}_{\lambda}^{n o r m^{2}}+\hat{\delta}_{\lambda}^{\text {norm }}{ }^{2}\right) .
\end{aligned}
$$

\footnotetext{
${ }^{13}$ For convenience, $\lambda^{s}$ is taken to be the implied value at the $99^{\text {th }}$ percentile of the full observed distribution.
} 
The final values of $(\hat{k}, \hat{\sigma})$ are taken to be those associated with the chosen value of $\lambda^{*}$, as shown in (18). ${ }^{1415}$ Sometimes, however, even this approach to smoothing may yield implausible results, as will be shown in an example in the next section of this paper

\subsection{Possibility of excluding regions from estimation}

As with the choice of $\lambda^{0 *}$, if the data to be fit are very strongly GPD, it would be desirable to use all the observations through the observed $100^{\text {th }}$ percentile. However, in survey data, the very top observed value is almost surely not the same as the population value. This is also likely to be the case for nearby observations. Thus, it is important at least to explore the sensitivity of the parameter estimates to variation in an upper truncation point for the support of the estimation, denoted as $\lambda^{T}$.

The $\mathrm{CH}$ approach has the additional benefit that it can easily be adapted to a situation where some part of the observed data is measured with error. In principle, the set of such data be in any part of the observed distribution. The aspect of this issue considered in this paper is the possibility where the very top of the observed distribution may be incomplete or corrupted, either because of non-random lack of observation, other effective under-coverage, or other measurement error. This possibility is addressed here in two ways: one by excluding some part of the observed data from the estimation, and the other by treating some part of the population as if it is not observed at all. This section addresses the former and the following section addresses the latter.

Because the $\mathrm{CH}$ approach depends only on pairs of values and corresponding empirical cumulative probabilities to compute the sets of estimates of $(\widehat{k}, \hat{\sigma})$, it is straightforward to exclude any set of observations of $\lambda$ (and the corresponding values of $p$ ) less than $\lambda^{T}$ below the observed $100^{\text {th }}$ percentile. However, to use the relationship in (11) in the first stage of selection of parameter values for a given $\lambda^{0}$ requires some adjustment to the test statistic.

The size of the region of the full observed distribution of $\lambda$ that is excluded is the distance $100-\Pi\left(\lambda^{T}\right)$ percentile points, where $\Pi\left(\lambda^{T}\right)$ is the empirical percentile of $\lambda^{T}$. The approximate adjustment adopted for the test statistic is to double the weight of the elements of (11) in the region from half this distance below $\Pi\left(\lambda^{T}\right)$ up to $\Pi\left(\lambda^{T}\right)$, and from $\lambda^{0 *}$ up the same distance. A comparable adjustment is made for (12).

One way to test the sensitivity of the estimation to exclusion of some extent of the upper tail is to compute $(\widehat{k}, \hat{\sigma})$ as described above, for a range of values of $\lambda^{T}$, and to compare the relative robustness of the parameter estimates. Formally, for $\lambda^{0 *}$ determined for a given value of $\lambda^{T}$, let $\operatorname{HCVM}\left(\lambda^{0 *}, \lambda^{T}\right)$ be the set values of the CVM statistic for $(\widehat{k}, \hat{\sigma})$ estimated for successively higher values of the location parameter, up to $\lambda^{T}$, as summarized in (19). Let $\operatorname{PCVM}\left(\lambda^{0 *}, \lambda^{T}\right)$ be the weighted percent of the elements of $\operatorname{HCVM}\left(\lambda^{0 *}, \lambda^{T}\right)$ that pass the CVM test, as shown in (20).

$$
\begin{gathered}
\operatorname{HCVM}\left(\lambda^{0 *}, \lambda^{T}\right)=\left\{\operatorname{CVM}(\lambda) \mid \lambda^{0 *} \leq \lambda<\lambda^{T}\right\} . \\
\operatorname{PCVM}\left(\lambda^{0 *}, \lambda^{T}\right)=\frac{\sum_{\lambda^{0 *} \leq \lambda<\lambda^{T}}\left(\operatorname{CVM}(\lambda) \leq \operatorname{TCVM}\left(\hat{k}_{\lambda}\right)\right) \operatorname{ESS}(\lambda)}{\sum_{\lambda^{0 *} \leq \lambda<\lambda^{T}} \operatorname{ESS}(\lambda)}
\end{gathered}
$$

\footnotetext{
${ }^{14}$ As implemented in the examples later in the paper, the selection in (18) is further constrained to imply that at least 50 observations would remain to support the estimation.

${ }^{15}$ Alternatively, one could simply use $\hat{k}_{\text {mean }}$ and $\hat{\sigma}_{\text {mean }}=\hat{\delta}_{\text {mean }} \hat{k}_{\text {mean }}$. The approach described in the body of the text turns out to be computationally more convenient, and the difference is minor.
} 
Repeat (19) and (20) for a range of candidate values of $\lambda^{T} \epsilon\left(\lambda_{1}^{T}, \lambda_{\max }^{T}\right)$, where $\lambda_{1}^{T}>\lambda^{*}$ and $\lambda_{\max }^{T} \leq$ $100 .{ }^{16}$ Choose as the final value of $\lambda^{T}$ the candidate value with the minimum value of PCVM.

\subsection{Compensating for the possibility that some very top group is effectively not covered}

It is possible that the maximum population values may more seriously misrepresent the extreme right tail than in the case discussed above, in the sense that some part of the extreme tail of the population may be altogether unobserved. In such a case, the empirical cumulative probabilities associated with each value would be biased upward. For example, if the true top one percent is not included at all, then the observed $100^{\text {th }}$ percentile is the true $99^{\text {th }}$ percentile, the observed $50^{\text {th }}$ percentile is the true $49.5^{\text {th }}$ percentile, etc. The structure of the $\mathrm{CH}$ method is also easily modified to allow for this possibility. If the population unobserved amounts to the highest $\rho$ percentiles of the true overall distribution, for a given $\lambda^{0 *}$ it is only necessary to reduce the empirical value of $P$ in the estimation by a factor of $\frac{\left(\left(100-\Pi\left(\lambda^{0 *}\right)\right) / 100\right)}{\left(\left(100-\Pi\left(\lambda^{0 *}\right)+\rho\right) / 100\right)}$ and to make a comparable adjustment in (11) and (12).

As yet, there is no clear formal criterion for selecting a specific value of $\rho$ and its corresponding set of estimates. For the two example data sets used in this paper, the data indicate that varying $\rho$ also causes the number of observations used to fit the GPD to vary, and within the range $\rho \leq 1$ considered, the size of the support has a maximum value. As a working hypothesis, maximizing the sample support is taken as a selection criterion in the empirical analysis in the following section.

\subsection{Conditioning estimates on a known total}

In some cases, external information may be available to give an exact value or sufficient approximation of the aggregate amount of $\lambda$ for its full distribution, say $\mathrm{A}_{0}$. The $\mathrm{CH}$ model can also be adapted in a straightforward way to incorporate such information. For $k>-1$, the implied mean of the amount remaining above a value of $\lambda_{i}$ for an associated remaining population of size $N_{\lambda_{i}}$ is given by

$$
\frac{A_{\lambda_{i}}}{N_{\lambda_{i}}} \equiv \mu_{\lambda_{i}}=\left(A_{0}-\sum_{\lambda<\lambda_{i}} W(\lambda) \lambda\right) / N_{\lambda_{i}} .
$$

Solving for $\delta$ as described in (8) and using the relationship expressed in (2), one may determine $(\widehat{k}, \hat{\sigma})$ as follows: ${ }^{17}$

\footnotetext{
${ }^{16}$ In the application here, only values of $\lambda^{T}$ within the observed top one percent are considered.

${ }^{17}$ One could also solve directly for $k$ by substituting $\sigma=\left(\mu_{\lambda_{i}}-\lambda_{i}\right)(1+k)$ into equation 1 and rearranging terms to derive the following equation with only $k$ unknown, as follows:

$$
1-p_{j}=\left(1-\frac{k \lambda_{j}}{\left(\mu_{\lambda_{i}}-\lambda_{i}\right)(1+k)}\right)^{1 / k}
$$

The estimate of $k$ could be determined by search. The estimate of $\sigma$ would be given by (23). One could also solve more directly for an estimate of $\delta$ by searching for the value of delta that solves the following equation:

$$
\left(\mu_{\lambda_{i}}-\lambda_{i}\right) \ln \left(1-p_{j}\right)=\left(\delta-\left(\mu_{\lambda_{i}}-\lambda_{i}\right)\right) \ln \left(1-\lambda_{j} / \delta\right)
$$
}

However, these alternatives appear to be empirically less robust. 


$$
\begin{gathered}
\hat{k}=\frac{\mu_{\lambda_{i}}-\lambda_{i}}{\hat{\delta}-\left(\mu_{\lambda_{i}}-\lambda_{i}\right)} \\
\hat{\sigma}=\left(\mu_{\lambda_{i}}-\lambda_{i}\right)(1+\hat{k}) .
\end{gathered}
$$

If some fraction of $A_{0}$ is not accounted for within the observed data, then the difference must be explained by understatement of the observed values, errors in weighting the observed values, values associated with an unobserved population, or a combination of these factors. In the following empirical section, the use of an aggregate value to constrain the GPD estimation is considered in the context of some extent of potentially unobserved population.

\section{Application}

\subsection{Description of the data used}

This section applies the technical apparatus of the previous section to data on net worth from the 2017 Austrian implementation of the Household Finance and Consumption Survey (HFCS) for Austria (see Fessler et al. [2019] and Albacete et al. [2019]) and the 2016 Survey of Consumer Finances (SCF) for the U.S. (see Bricker et al. [2017] and Kennickell [2017]). ${ }^{18}$ The larger HFCS project consists of ex ante harmonized surveys for 18 euro-area countries and two other European countries. Both the HFCS and the SCF are specifically designed to collect wealth data, ${ }^{19}$ and both employ many similar technical approaches. ${ }^{20}$ The wealth measure used here is one focused on assets and liabilities over which the household has direct ownership or responsibility; it excludes contingent assets, such as the value of future pension payments.

The critical dimension of difference between the surveys is the approach to sampling. The Austrian HFCS uses a nationally representative geographically based sample design, with some over-sampling of Vienna intended to offset expected higher unit nonresponse there. The overall response rate in 2017 was 49.8. The SCF uses a two-part sample design. One part is a geographically based sample similar to that used for the Austrian survey; this sample had a response rate of about 65 percent. The other part is a list sample selected from statistical records derived from individual income tax returns, which is stratified and disproportionately sampled in order to focus most sharply on the wealthiest households; the response rate in the list sample ranged from about 12 percent for households in the highest stratum, to about 56 percent in the least wealthy stratum. The two SCF samples are combined through a weighting process. Note that the list sample explicitly excludes the set of people identified by Forbes magazine as the 400 wealthiest people in the U.S. The public version of the data, which is used here, also excludes the survey observations that have wealth greater than or equal to the lowest wealth value for the Forbes population.

The results of the two surveys also differ in the extent to which they align with external wealth aggregates from national accounts. For example, the Austrian survey understates a national account measure of aggregate net worth, by about a quarter in 2017; in contrast, the SCF time series tracks aggregate wealth

\footnotetext{
${ }^{18}$ Except where explicitly noted, no attempt has been made to convert the monetary values reported in this paper to a common currency. For reference, the exchange rate as of January 2, 2017 was 1.05 USD per euro.

${ }^{19}$ In this paper, the terms wealth and net worth are used synonymously.

${ }^{20}$ Of particular relevance here, both surveys use multiple imputation for missing data, and both provide a set of replicate survey weights to be used for estimating sampling variability of estimates. These technical devices have the effect of multiplying the distinct view of each data set -5 times to account for the multiple imputation implicates and about 1,000 times to account for the sample replicates. Many of the calculations reported here require an exceptionally large amount of time and displaying comparable results for or based on all the views of the data considered would require substantial space. Thus, except where specifically noted, the results reported are those based on the first implicate of each survey.
} 
much more closely (see Henriques et al. [2014] and Batty et al. [2019]). ${ }^{21}$ These differences between the two surveys are believed to be explained at least in large part by differences in the effective coverage of the upper tail of the wealth distribution, most likely as a result of nonrandom unit nonresponse. The key advantage the SCF has in this regard is its ability to use the linkage with tax-based data in adjusting the survey for nonresponse. In contrast, the Austrian survey must address nonresponse with a set of characteristics much less correlated with wealth. The SCF also has many more observations to represent the upper tail. For example, in the Austrian HFCS, 29 observations represent the wealthiest one percent, whereas in the SCF, there are 681 such observations.

Table 1: Descriptive statistics for 2017 Austrian HFCS and 2016 SCF. ${ }^{1}$

\begin{tabular}{|c|c|c|}
\hline Item & 2017 Austrian HFCS & $2016 \mathrm{SCF}$ \\
\hline Mean $^{2}$ & 237500 & 689200 \\
\hline & $11200 \quad \mathbf{1 8 4 0 0}$ & 7100 \\
\hline Median $^{2}$ & 74800 & 97300 \\
\hline & 1180 & 2700 \\
\hline Share top $1 \%$ & 22.9 & 38.6 \\
\hline Gini coefficient & $\begin{array}{r}3.01 \\
0.723\end{array}$ & $\begin{array}{l}0.16 \\
0.850\end{array}$ \\
\hline P90-P25 ratio & $\begin{array}{rr}0.011 & \mathbf{0 . 0 1 9} \\
44.9 & \end{array}$ & $\begin{array}{cc}0.001 & \mathbf{0 . 0 0 3} \\
116.8 & \end{array}$ \\
\hline $\begin{array}{l}\text { Number of } \\
\text { observations }\end{array}$ & 0.66 & $1.75 \quad \mathbf{4 . 8 6}$ \\
\hline \multicolumn{3}{|c|}{$\begin{array}{l}\text { 1. The reported values were calculated using all implicates and the sample } \\
\text { replicates. } \\
2 \text {. The mean and median figures are given in the home currency in each case. For } \\
\text { reference, the exchange rate as of January } 2,2017 \text { was } 1.05 \text { USD per euro. Using } \\
\text { this rate, the SCF mean in euro was } 656400 \text { and the median was } 92700 \text {. } \\
\text { Note: The number given in italics below each main estimate is the estimated } \\
\text { standard error with respect to imputation, and the number given in bold is the } \\
\text { estimated standard error with respect to both imputation and sampling. }\end{array}$} \\
\hline
\end{tabular}

The surveys differ substantially in terms of their levels and other distributional characteristics (table 1). Adjusted for the difference in currencies, median Austrian household wealth measured in the HFCS was about 81 percent of the level observed in the SCF, but the Austrian mean is only about 36 percent of the SCF value. ${ }^{22}$ This indication of greater wealth concentration observed in the SCF is reinforced by the very different values of the Gini coefficient and the shares of the wealthiest one percent.

Reflecting in part the much lower sampling rate at the top of the distribution in the Austrian HFCS, the value of the main statistics given in the table have much greater total variability than the SCF estimates. For example, in the case of the share of the top 1 percent, the total standard error relative to the estimate is

\footnotetext{
${ }^{21}$ It is possible in some cases the shortfall of survey estimates of wealth relative to corresponding aggregates may be explained in part by under-reporting of values across the wealth distribution or in parts with disproportionately large or small values. To focus more strongly on the model presented in this paper, that possibility is ignored here.

${ }^{22}$ Some of the difference in levels is explained by the much lower rate of homeownership in Austria than in the U.S.; Austria has a much higher proportion of social housing. Such differences - and indeed the very question of what a measure of net worth should include - are important, but beyond the scope of this paper.
} 
almost 12 times larger. Note that for both sources, imputation variability is a substantial fraction of the total standard error for each statistic — around 60 percent for the mean.

The two distributions show a roughly similar profile of the percentage change (on a per percentile basis) in the level of wealth across the wealth distribution (figure 3 ). ${ }^{23}$ Both show a pattern of declining rates of increase from the point where wealth becomes positive, a flattening of the rate across the middle of the distribution, and a pattern of increase in the upper region of the distribution, that appears at least superficially consistent with some form of exponential distribution.

Figure 3: Percent change in net worth per percentile, 2017 Austrian HFCS and 2016 SCF.

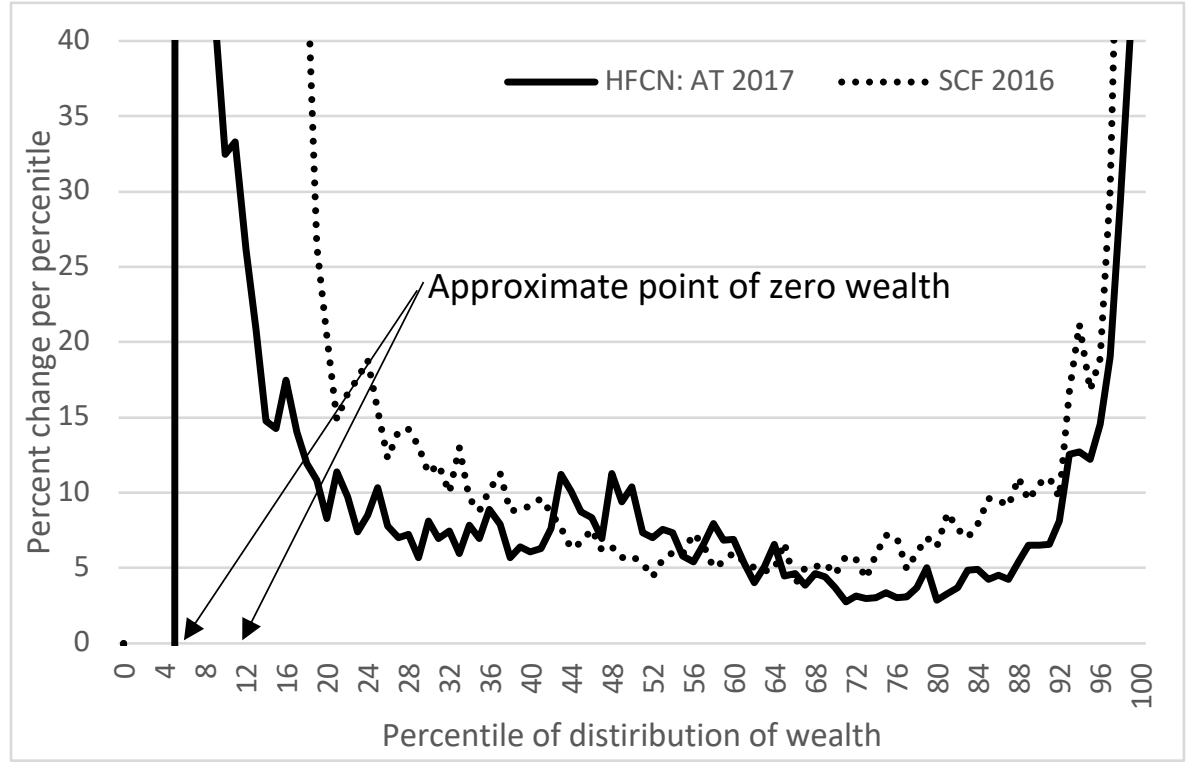

The linear relationship between the level of net worth and the mean of the exceedances from that wealth level, as expressed in (3), provides more insight into the specific question of whether the data are plausibly GPD. Note that there is good reason to think that even if the underlying empirical relationship is strictly GPD, the plot would show a line that progressively curves below a hypothetical straight line. The reason is that with a finite set of data, there is some limit to the highest wealth values observed, and the higher the level of net worth in the observed data, the more its associated mean of exceedances is affected by the "missing" extreme outer tail. The highest level of net worth observed necessarily has a zero mean of exceedances.

For both the Austrian HFCS (figure 4) and the SCF (figure 5), the data show the expected noisy pattern and a decline at the top of the wealth range. Both also show a reasonably linear pattern consistent with the GPD, up to about the $99^{\text {th }}$ percentile for the Austrian data and about the $98^{\text {th }}$ percentile for the SCF. Although both exhibit a sort of "notch" at about this point, the SCF data additionally show a subsequent pronounced approximately linear region, perhaps because of the greater density of sample observations for the SCF in this range. This difference appears to be reflected to a degree in the analysis that follows.

${ }^{23}$ The vertical lines represent the approximate point where the value of net worth is zero. The regions to the left of those points have been suppressed; in those regions, the positive wealth change divided by negative wealth yields a negative percentage change. The range of change above 40 percent has also been suppressed to show the pattern for the bulk of the distribution more clearly; for the Austrian HFCS (SCF) the percentage change from the $99^{\text {th }}$ to the $100^{\text {th }}$ percentile is 3,297 percent $(16,631$ percent $)$. 
Figure 4: Scatterplot of mean exceedances at levels of net worth (euro) from the 60th to 100th percentiles, 2017 Austrian HFCS.

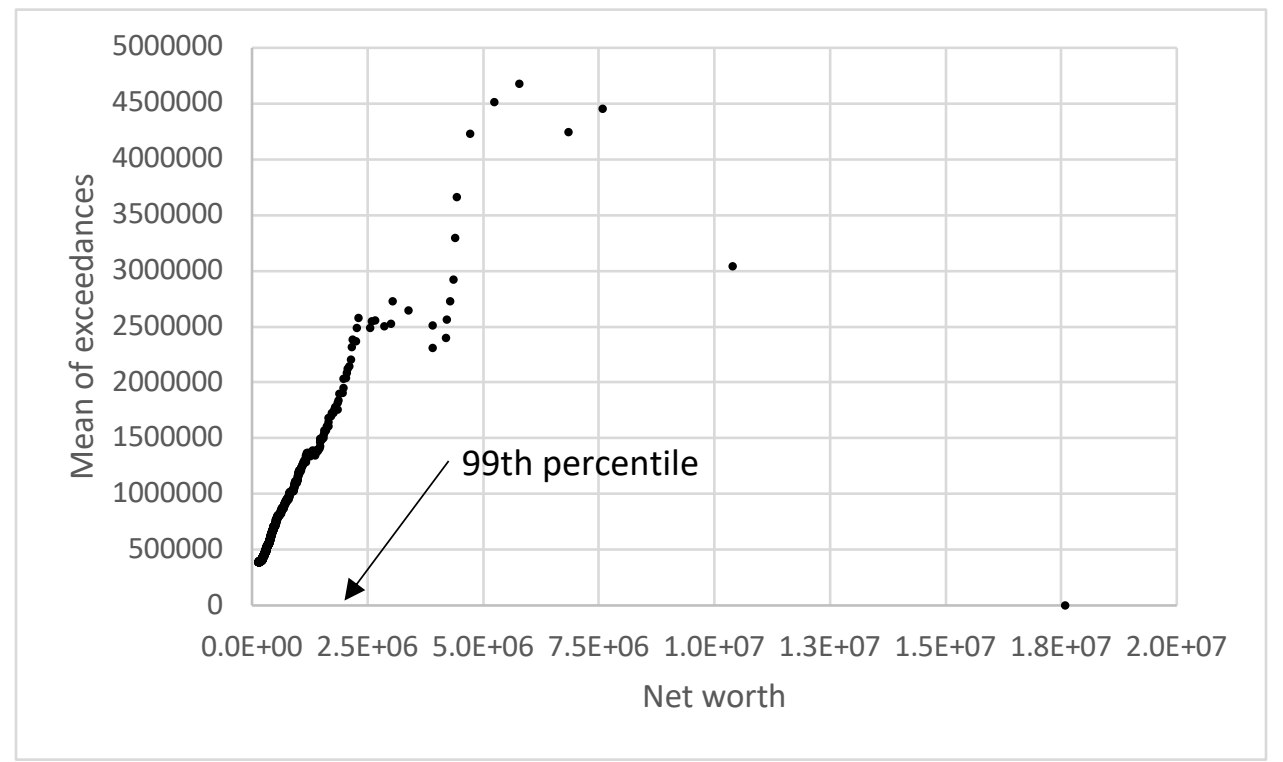

Figure 5: Scatterplot of mean exceedances at levels of net worth (USD) from the 60th to 100th percentiles, 2016 SCF.

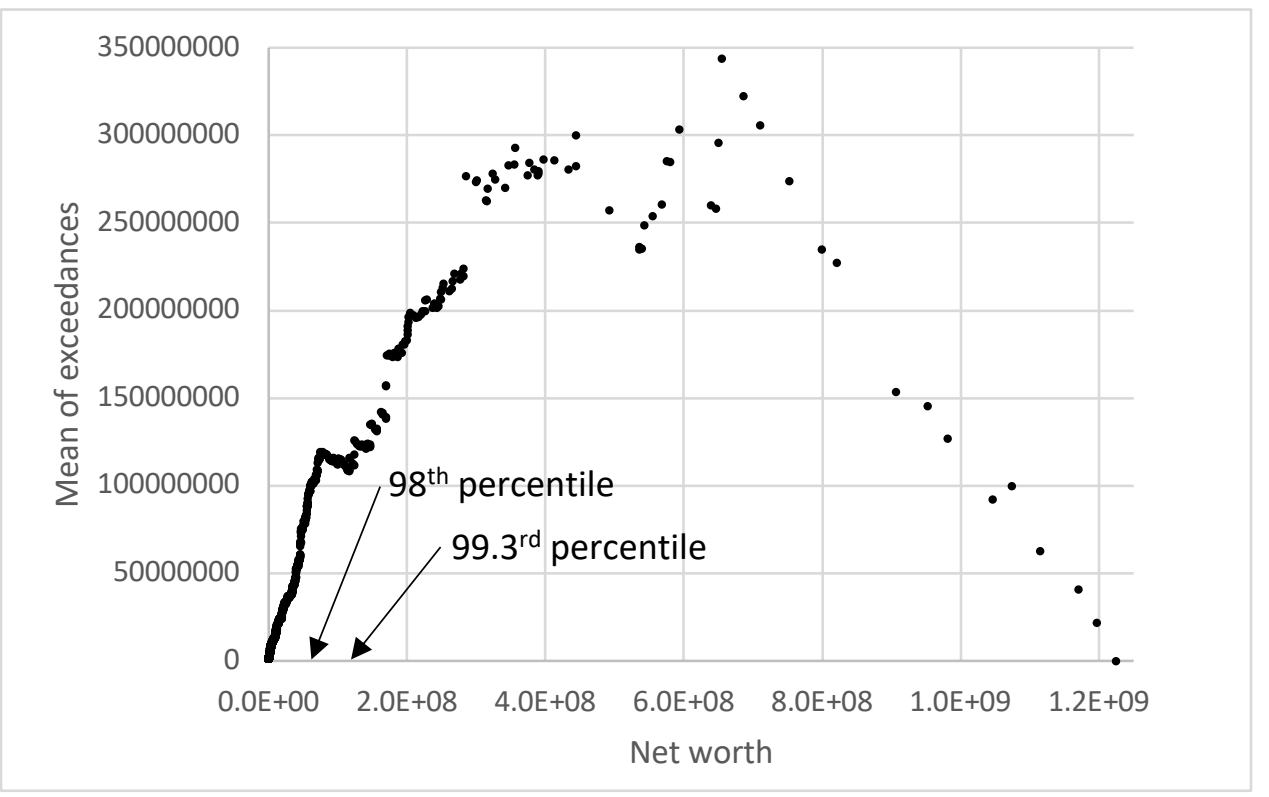

\subsection{Estimation, basic model}

The modified $\mathrm{CH}$ estimation method as implemented here yields about 5,100 candidate estimates for $(\widehat{k}, \hat{\sigma})$ for a given value of $\lambda^{0} .{ }^{24}$ For each of the two surveys, figure 6 shows a scatterplot of these

${ }^{24}$ During the course of developing the model and extensions presented in this paper, a large number of simulations were run to test whether the model could recover the correct GPD from appropriately altered data from a GPD with known parameters. Those tests were successful. However, it is one matter to be able to fit data with a known 
estimates for the first value of $\lambda^{0 *}$ that passes the CVM test. The estimates selected are shown as large white circles. Clearly, the range of the shape parameter $k$ is very wide, including some values (those less than -1 or greater than 0 ) that are inconsistent with the data more broadly. ${ }^{25}$ The spread of the estimates of $\hat{\sigma}$ is much less broad in the region of the final estimates, but spreads out notably both above and below that point, particularly so in the case of the SCF. Because this implementation of the $\mathrm{CH}$ method exploits so many data intervals, it is likely to discover whatever irregularities are present. In general, the spread of the parameter estimates underscores the importance of the criteria for the selecting the final estimates.

The solid line through each mass of data points represents the locus of points in $k, \sigma$ space that imply (according to (2)) the same mean of exceedances relative to the value of $\lambda^{0 *}$ for each of the surveys. Points below the lines imply higher values of that mean. That is, such points imply greater mass in the distribution above $\lambda^{0 *}$. Variations across that locus may imply very different degrees of concentration in the extreme of the upper tail.

Figure 6: Scatter plot of candidate values for $\widehat{k}$ and $\widehat{\sigma}$ for lowest value of $\lambda^{0 *}$ passing the CVM test, Austrian HFCS and SCF.

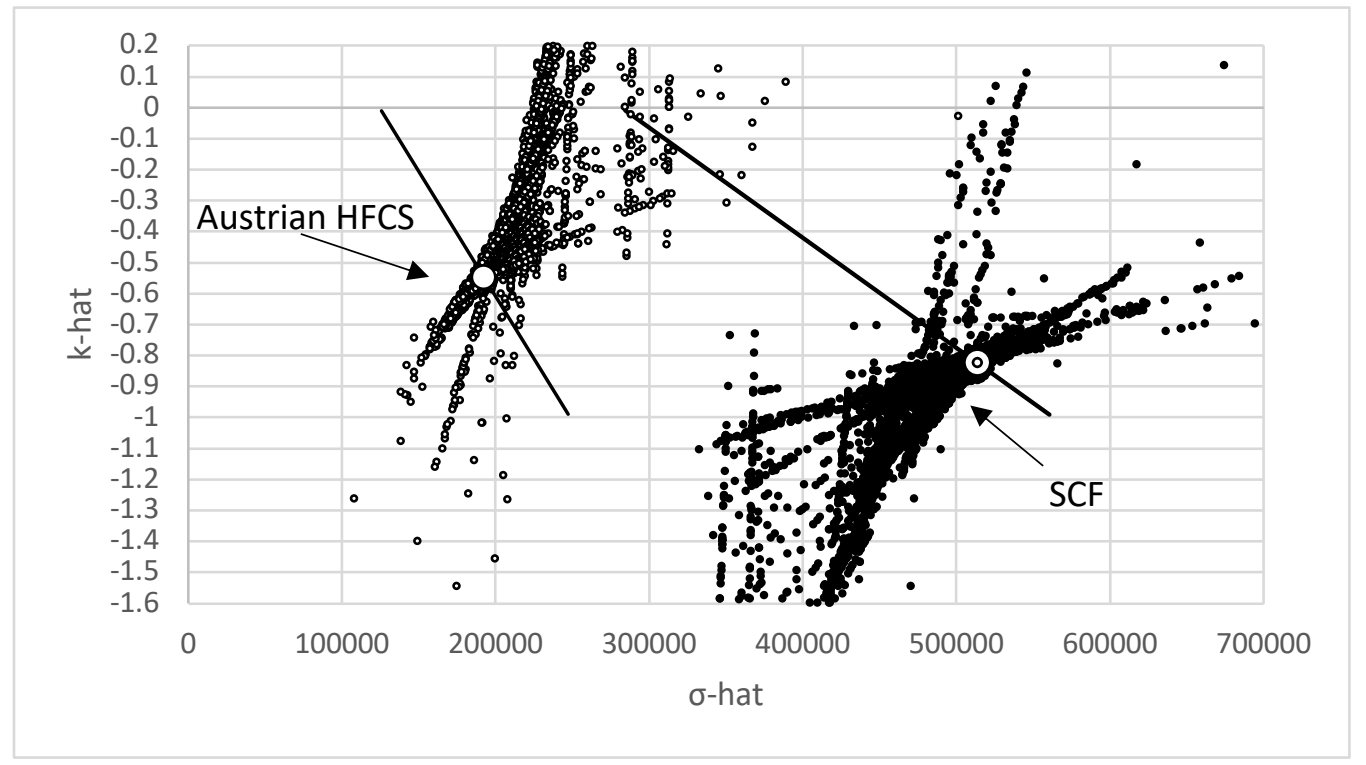

As noted in the methodology section, finding the lowest value for $\lambda$ that yields GPD parameters estimates that pass the CVM test does not necessarily guarantee that all higher values for $\lambda$ would also pass that test, or that the shape parameter and the scale parameter, suitably adjusted as in (3), would be even approximately the same. Figures $7 \mathrm{a}$ and $7 \mathrm{~b}$ show, for the Austrian HFCS and the SCF respectively, (ordered from top to bottom of the figures at the $75^{\text {th }}$ percentile) the Gini coefficient, the share of wealth attributed to the top 1 percent, the value of the CVM test, and the estimate of $\hat{k}$, all for a range of values of $\lambda$ taken as $\lambda^{0}{ }^{26}$ For convenience of comparison, these four plots are shown with an X-axis scaled in

distribution and quite another to fit empirical data of unknown distribution. Formal tests - and inspection for plausibility of outcomes - take on a high order of importance in the latter case.

${ }^{25}$ The full extent of the range is truncated in the figure in order to make the patterns of majority of the points more easily viewable.

${ }^{26}$ The appendix B gives the formulas for computing the distributional statistics reported. 
terms of the percentiles of the observed distribution, rather than the actual values of $\lambda$. Values for estimates that pass the CVM test are shown as solid lines.

Figure 7a: Gini coefficient, share of top 1 percent, CVM test value and $\widehat{k}$, by full-distribution percentile corresponding to $\lambda$, Austrian HFCS.

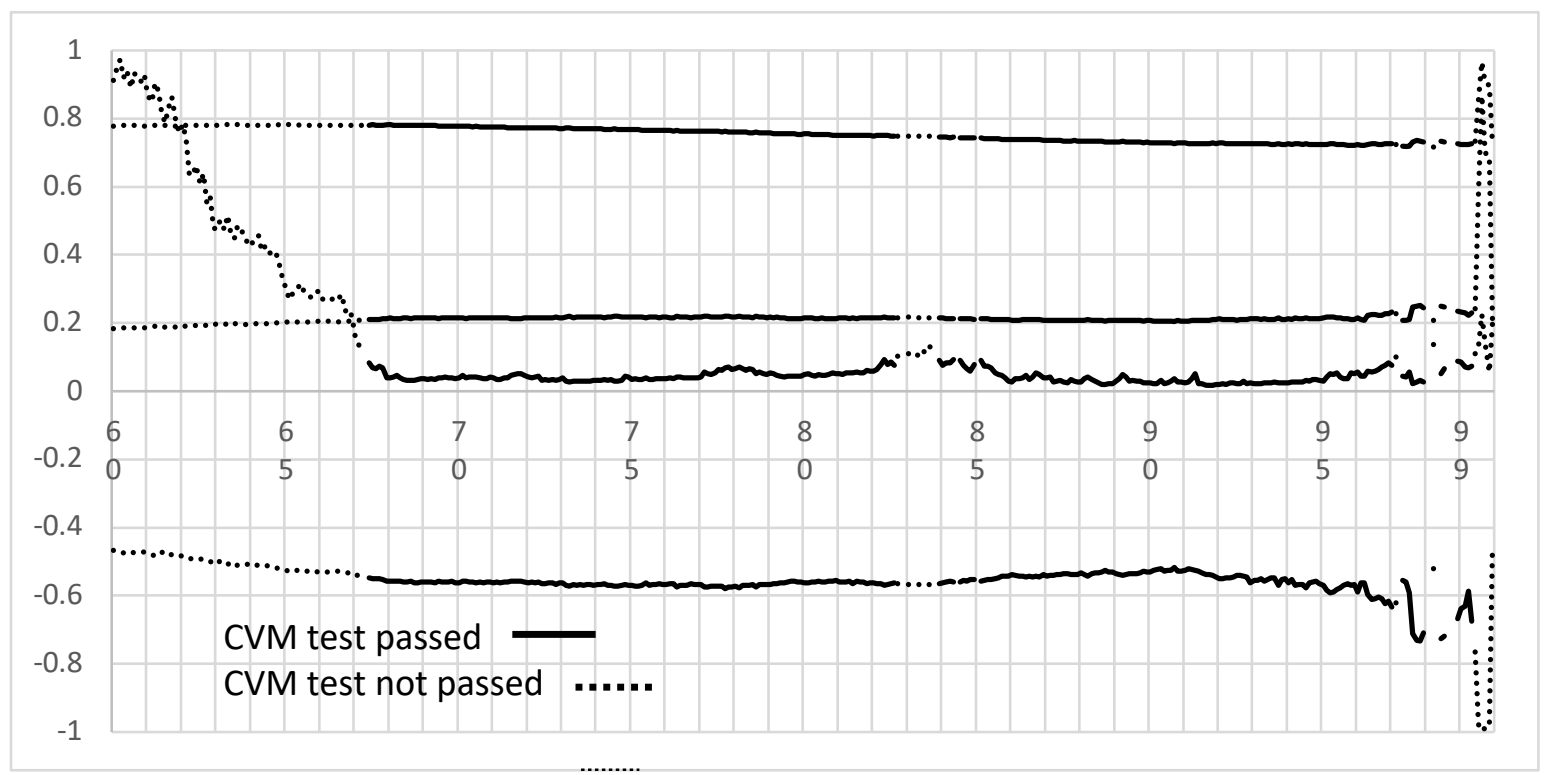

Figure 7b: Gini coefficient, share of top 1 percent, CVM test value and $\widehat{k}$, by full-distribution percentile corresponding to $\lambda$, SCF.

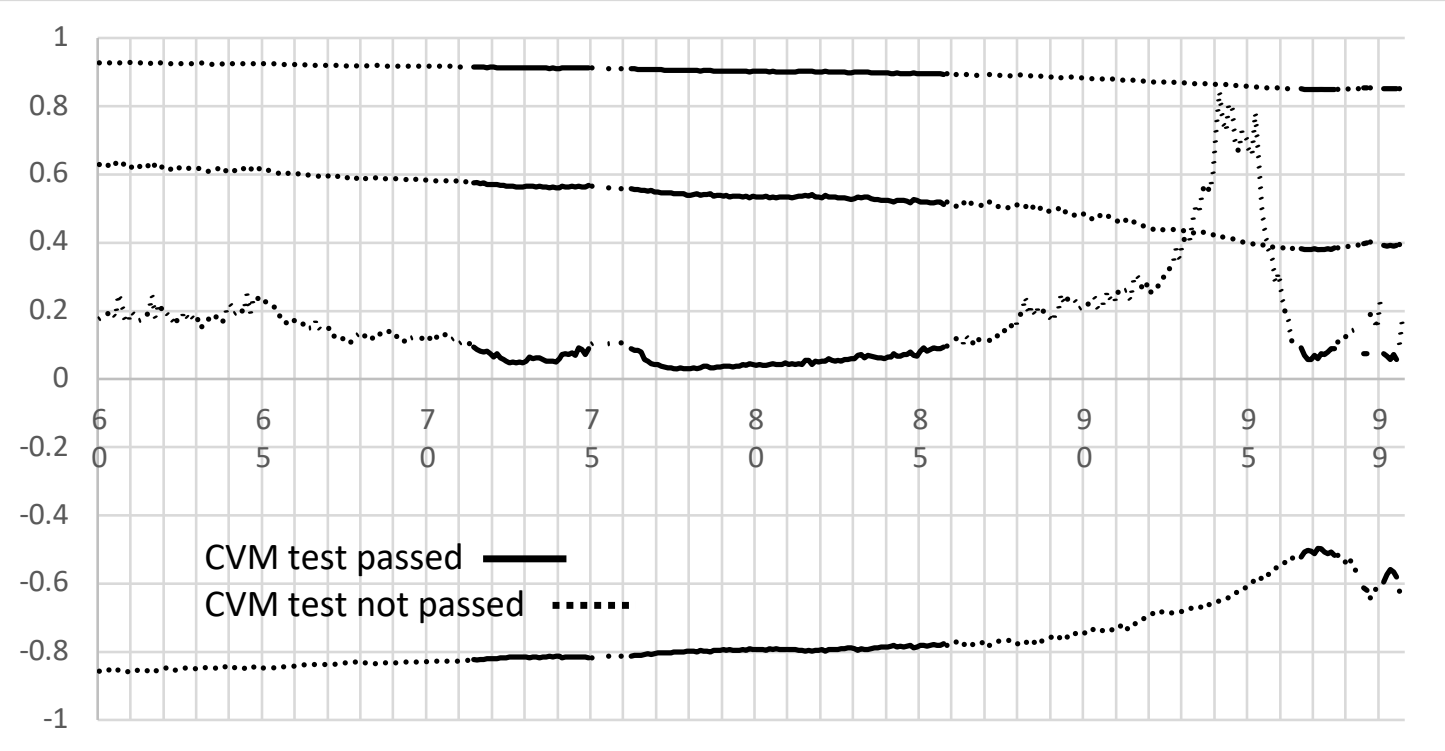

The most striking contrast between the Austrian HFCS and SCF results is the much greater variation in the four variables in the case of the SCF, particularly within the observed top decile of the wealth distribution. Once past an initial region that does not pass the CVM test, the Austrian data exhibit only a few relatively small irregularities until the top of the distribution. In the regions where the CVM test holds, the values of $\hat{k}$ and the top share are fairly steady. In the case of the SCF, however, the values of $\hat{k}$ and the top share tend to decline across the percentiles, and the values are notably different after the large 
jump in the CVM test values after about the observed $91^{\text {st }}$ percentile of $\lambda .{ }^{27}$ Moreover, whereas the Austrian HFCS values for the top share approximate the observed share, the SCF values below the $91^{\text {st }}$ percentile are substantially and implausibly higher than the observed value.

Table 2 reports three sets of estimates for the two data sets at this stage: (1) those for the smallest value of $\lambda$ for which the CVM test is passed, (2) those for $\left(\hat{k}_{\text {mean }}, \hat{\sigma}_{\text {mean }}\right)$ estimated using data over the range of $\lambda$ above the lowest value of $\lambda^{0 *}$, and (3) those for $\left(\hat{k}_{\text {mean }}, \hat{\sigma}_{\text {mean }}\right)$ estimated using only the region above the point of disruption in the CVM test values as noted above.

For the Austrian data, the differences in the estimates of $\hat{k}$ and the adjusted $\hat{\sigma}$ are relatively small across the three sets, and the estimates of the top share are about equal to the value of 21.3 percent for the first implicate. Moreover, the population mean net worth implied by the estimates (together with the data below the relevant value of $\lambda$ also varies little. ${ }^{28}$ While this degree of consistency may seem comforting, it is not enough for broader purposes of distributional modeling if these straightforward estimates cannot sufficiently replicate key aspects of the wealth distribution known from other sources. In particular, Austrian aggregate data on household net worth (see Oesterreichische National Bank [2019]), which are generally assumed to be reasonably comparable and reliable, suggest that mean wealth (and, necessarily, total net worth) is understated in the survey data even when augmented by the GPD tail, by about 26 percent. ${ }^{29}$ Thus, this basic approach to tail estimation appears to be inadequate in the case of the Austrian data.

While the estimates of the adjusted $\hat{\sigma}$ are also somewhat stable across the three sets of estimates for the $\mathrm{SCF}, \hat{k}$ and the other estimates differ greatly. Moreover, both the share estimates for the initial value of $\lambda^{0 *}$ and for $\lambda^{0 *}$ corresponding to $\hat{k}_{\text {mean }}$ are implausibly high: 57.4 percent for the former and 53.1 percent for the latter. In contrast, the 38.3 percent share estimate for $\lambda^{0 *}$ corresponding to $\hat{k}_{\text {mean }}$ above the top break is close to the 38.6 percent figure observed directly from the data, and the estimated population mean similarly approaches the directly observed measure. ${ }^{30}$ However, comparison with a population mean derived from aggregate data is less straightforward. As Batty et al. [2019] show, making a conceptual alignment between the SCF data and the aggregate accounts requires a variety of adjustments (including an imputation for the value of defined-benefit pensions) that are well beyond the scope of this paper. Nonetheless, the strong message from that paper is that, suitably adjusted, the SCF does very well in representing total net worth and its major sub-components. As noted earlier, the very wealthiest people in the U.S. are deliberately excluded from the SCF, and their wealth is about 4.2 percent of the total wealth captured in the public version of SCF used here. For the expository purposes of this paper, the SCF measure of net worth augmented by this additional percent is taken to be a sufficient proxy for a comparable aggregate-based measure of net worth.

\footnotetext{
${ }^{27}$ Note that this jump reflects, in part, the fact that the parameters resulting from the right-tail Anderson-Darling criterion are not constrained to be within the set of values passing the CVM test, since that set is null in this region. However, the result is not an artifact of the use of the right-tailed Anderson-Darling statistic to select the candidate values; using instead the estimates with the minimum value of the CVM test yields a very similar result.

${ }^{28}$ As shown in appendix table A1, the model-supplemented estimates also yield notably smaller confidence intervals for the estimates of the overall mean and the share of the top 1 percent.

${ }^{29}$ One may question the extent to which the aggregate wealth value is exactly comparable and reliable, but it is taken as a sufficient approximation for the purposes of this paper.

${ }^{30}$ Note that this estimate is supported by 1042 observations, reflecting the strong degree of oversampling of the wealthy in the SCF. However, shown in appendix table A1, the confidence intervals for both the overall mean and the share of the top 1 percent are larger than those based on the unadjusted data.
} 
Table 2: Parameter estimates and related statistics, for various values of $\lambda^{0 *}$, Austrian HFCS and SCF.

\begin{tabular}{|c|c|c|}
\hline & Austrian HFCS & SCF \\
\hline Initial $\lambda^{0 *}$ & 216500 & 304370 \\
\hline Percentile & 67.4 & 71.4 \\
\hline$\hat{k}$ & -0.548 & -0.823 \\
\hline$\hat{\sigma}$ & 193233 & 514681 \\
\hline$\hat{\sigma}$ adjusted to $99^{\text {th }}$ percentile ${ }^{\mathrm{a}}$ & 1254102 & 8725944 \\
\hline Share of top 1 percent & 21.1 & 57.4 \\
\hline Gini coefficient & 0.7812 & 0.915 \\
\hline Implied population mean & 244050 & 945045 \\
\hline Number of observations $>\lambda^{0 *}$ & 830 & 2690 \\
\hline$\lambda^{0 *}$ corresponding to $\hat{k}_{\text {mean }}$ & 320000 & 499250 \\
\hline Percentile & 79.5 & 79.9 \\
\hline$\hat{k}_{\text {mean }}$ & -0.560 & -0.794 \\
\hline$\hat{\sigma}$ & 244235 & 720671 \\
\hline$\hat{\sigma}$ adjusted to $99^{\text {th }}$ percentile ${ }^{\text {a }}$ & 1268739 & 8493279 \\
\hline Share of top 1 percent & 21.4 & 53.5 \\
\hline Gini coefficient & 0.756 & 0.902 \\
\hline Implied population mean & 245240 & 890595 \\
\hline Number of observations $>\lambda^{0 *}$ & 527 & 2247 \\
\hline $\begin{array}{l}\lambda^{0 *} \text { corresponding to } \\
\hat{k}_{\text {mean }} \text { above top break }\end{array}$ & 726000 & 3182800 \\
\hline Percentile & 93.6 & 96.6 \\
\hline$\hat{k}_{\text {mean }}$ & -0.547 & -0.523 \\
\hline$\hat{\sigma}$ & 473320 & 3988104 \\
\hline$\hat{\sigma}$ adjusted to $99^{\text {th }}$ percentile ${ }^{\text {a }}$ & 1253390 & 7699098 \\
\hline Share of top 1 percent & 21.1 & 38.3 \\
\hline Gini coefficient & 0.724 & 0.851 \\
\hline Implied population mean & 244182 & 671207 \\
\hline Number of observations $>\lambda^{0 *}$ & 167 & 1042 \\
\hline Observed values for $1^{\text {st }}$ implicate & & \\
\hline Share of top 1 percent & 21.3 & 38.6 \\
\hline Gini coefficient & 0.717 & 0.848 \\
\hline Sample mean & 245151 & 679847 \\
\hline Population mean ${ }^{\mathrm{c}}$ & 332692 & 708536 \\
\hline \multicolumn{3}{|c|}{$\begin{array}{l}{ }^{\mathrm{a}} \text { The value of net worth at the } 99^{\text {th }} \text { percentile and the value of } \hat{k} \text { are } \\
\text { used to adjust } \hat{\sigma} \text { to the implied value at the } 99^{\text {th }} \text { percentile. } \\
{ }^{\mathrm{b}} \text { The top break taken for the Austrian HFCS is the } 85^{\text {th }} \text { percentile and } \\
\text { that for the SCF is the } 95^{\text {th }} \text { percentile. } \\
{ }^{\mathrm{c}} \text { For Austria, the population mean is taken to be the aggregate value } \\
\text { divided by the number of households. See the main text for an } \\
\text { explanation of the U.S. population mean. } \\
\text { Note: Appendix B provides the calculations to derive the share of the } \\
\text { top } 1 \text { percent and the Gini coefficient. }\end{array}$} \\
\hline
\end{tabular}

In summary, the wealth data from both surveys show formal signs of being plausibly describable as GPD for a broad range of the wealth distribution. But the Austrian data seriously understate aggregate wealth under all three sets of estimates, while the SCF greatly overstates aggregate wealth for the first two and 
comes close to the aggregate with the estimates in the third, where the estimates are focused more sharply on the top of the distribution. What explains this pattern: Is the extreme upper tail simply drawn from a GPD with a different set of parameters, and in the Austrian case there are insufficient observations to identify those parameters? Is the observed distribution distorted by reporting errors, coverage errors or some combination of the two? The following to sections attempt to address these questions and investigate the extent to which external information can be brought to bear in developing estimates that align with the aggregate data, yet still apply to a substantial range of the wealth distribution.

\subsection{Estimation, allowing for data imperfections}

The method described earlier for omitting data regions from the estimation can be used to explore the sensitivity of the estimates to potentially erroneous or overly noisy data. To focus on the effects of reporting errors at the top of the distribution, table 3 shows the estimates given in table 2, recomputed using various data exclusions limits: above the $99^{\text {th }}$ and $98^{\text {th }}$ percentiles for the Austrian data and above the $99^{\text {th }}$ and $99.5^{\text {th }}$ percentiles for the SCF. ${ }^{31}$

For the Austrian data, omitting the top one percent has a relatively small effect on the first two sets of estimates; it noticeably reduces the overall mean and down-weights the upper tail for the third set. Whereas omitting data for the top two percent increases both the overall estimated mean and the top share in all three cases, especially for the third set of estimates. For the SCF, omitting the top 0.5 percent further increases the already implausible estimated overall mean and top share for the first two sets of estimates; omitting the top one percent increases these values for all three sets of estimates. Given, this degree of variation in outcomes for relatively small changes in the basis of the estimation and the inconsistency of the results, it appears that data trimming, at least taken alone, is unlikely to improve the performance of the estimation. ${ }^{32}$

\subsection{Estimation, allowing for an unobserved extreme upper tail}

Alternatively, it may be that the values reported in the top of the observed distribution are not erroneous, but they are misplaced in the distribution, because the upper tail is not captured in the data at all above some point. Because it is highly unlikely in the case of the Austrian data and impossible in the case of the SCF that the value at the $100^{\text {th }}$ percentile is the true population maximum, allowing for at least some degree of missing mass at the top of the distribution seems appropriate.

To give an indication of the sensitivity of the estimates to an unobserved tail, for both data sets, the top 0.1 and the top 0.5 percent were considered to have been unobserved. In addition, for the SCF, a percent equivalent to the 400 people in the Forbes list of the purportedly wealthiest Americans was treated as unobserved, as is known to be the case.

As shown in table 4, for the Austrian data under the assumption of an unobserved 0.1 percent, there is an implied small increase in the top share, the Gini coefficient and the population mean. Treating the top one-half percent as unobserved implies a more substantial increase in these measures, though the mean is still only about 80 percent of the value implied by the aggregate wealth figure. The relationship does not appear to be necessarily monotonic in the extent of the missing mass assumed: if the wealthiest one percent is assumed not to be observed at all (not shown in the table), these measures fall to a level between the two scenarios shown in the table. For the SCF estimates, although explicitly recognizing the

\footnotetext{
${ }^{31}$ For the Austrian data, 53 observations were excluded beyond the $98^{\text {th }}$ percentile and 28 observations beyond the $99^{\text {th }}$ percentile; the comparable figures for the SCF were 680 observations above the $99^{\text {th }}$ percentile and 563 above the $99.5^{\text {th }}$ percentile. A variety of other intervals were also tested but did not yield additional insights.

${ }^{32}$ For this reason, the statistic given by (18) for choosing a cut-off point is not reported here.
} 
omission of the 400 wealthiest people makes virtually no difference, treating the top 0.1 percent or the top 0.5 percent as unobserved implies substantial differences. However, as with the other SCF GPD-based estimates presented to this point, the first two sets of estimates can be rejected as implausible. For the third set, treating the top 0.1 percent as unobserved yields a top share of 39.1 percent; this is higher than the unadjusted estimate, but still about a percent below (but within the confidence interval of) the comparable 40.3 percent point estimate of Bricker et al. [2019] made incorporating a simple Pareto extrapolation of SCF data and the observations captured in the Forbes data. Estimates treating the wealthiest 0.5 percent as unobserved imply a slightly lower top share and an implied mean greater than the assumed population mean; a point between 0.1 and 0.5 percent would align with that mean. Thus, it appears that as in the case of the exclusion of some part of the upper tail from the estimation support, this approach alone is not sufficient to provide a reliable basis for extrapolating the observed data for the implied total wealth to come close to the aggregate figure.

Table 3: Parameter estimates and related statistics, for various values of $\lambda^{0 *}$, Austrian HFCS and SCF, where data above various percentiles have been truncated for purposes of estimation.

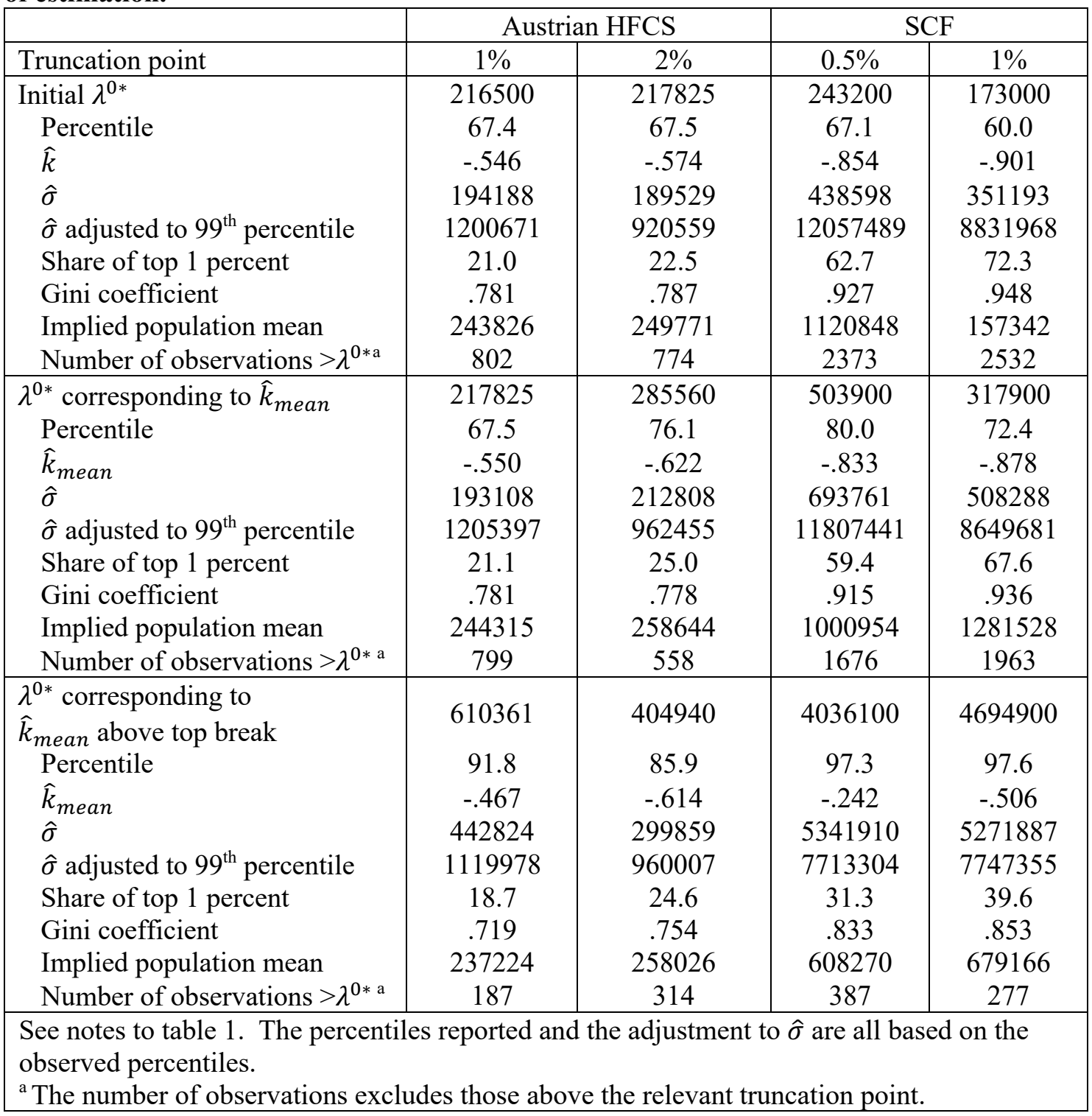


Table 4: Parameter estimates and related statistics, for various values of $\lambda^{0 *}$, Austrian HFCS and SCF, where some extent of the top of the distribution is unobserved.

\begin{tabular}{|c|c|c|c|c|c|}
\hline Survey & Austria & Austria & SCF & SCF & SCF \\
\hline Missing mass & $0.1 \%$ & $0.5 \%$ & $0.00032 \%$ & $0.1 \%$ & $0.5 \%$ \\
\hline Initial $\lambda^{0 *}$ & 216500 & 222100 & 304370 & 246900 & 277710 \\
\hline Percentile & 67.4 & 67.9 & 71.4 & 67.5 & 69.6 \\
\hline$\hat{k}$ & -.562 & -.639 & -.824 & -.847 & -.903 \\
\hline$\hat{\sigma}$ & 191872 & 182955 & 514382 & 450509 & 464950 \\
\hline $\begin{array}{l}\hat{\sigma} \text { adjusted to } 99^{\text {th }} \\
\text { percentile }\end{array}$ & 1279426 & 1415585 & 87733358 & 8955029 & 9503548 \\
\hline Share of top 1 percent & 21.8 & 26.8 & 57.5 & 61.5 & 73.0 \\
\hline Gini coefficient & .785 & .804 & .915 & .925 & .949 \\
\hline $\begin{array}{l}\text { Implied population } \\
\text { mean }\end{array}$ & 248465 & 271369 & 969016 & 1090362 & 1626863 \\
\hline $\begin{array}{l}\text { Number of observations } \\
>\lambda^{0 *}\end{array}$ & 830 & 818 & 2690 & 2868 & 2771 \\
\hline $\begin{array}{l}\lambda^{0 *} \text { corresponding to } \\
\hat{k}_{\text {mean }}\end{array}$ & 258263 & 240000 & 488410 & 448420 & 438030 \\
\hline Percentile & 73.0 & 70.6 & 79.6 & 78.3 & 77.9 \\
\hline$\hat{k}_{\text {mean }}$ & -.575 & -.628 & -.794 & -.809 & -.880 \\
\hline$\hat{\sigma}$ & 206984 & 193278 & 714575 & 671751 & 631082 \\
\hline $\begin{array}{l}\hat{\sigma} \text { adjusted to } 99^{\text {th }} \\
\text { percentile }\end{array}$ & 1294695 & 1392736 & 8482480 & 8631405 & 9290340 \\
\hline Share of top 1 percent & 22.2 & 25.8 & 53.4 & 55.7 & 68.1 \\
\hline Gini coefficient & .774 & .785 & .903 & .908 & .936 \\
\hline $\begin{array}{l}\text { Implied population } \\
\text { mean }\end{array}$ & 248832 & 269452 & 882503 & 897764 & 1296370 \\
\hline $\begin{array}{l}\text { Number of observations } \\
>\lambda^{0 *}\end{array}$ & 687 & 749 & 2263 & 2335 & 2355 \\
\hline $\begin{array}{l}\lambda^{0 *} \text { corresponding to } \\
\hat{k}_{\text {mean }} \text { above top break }\end{array}$ & 669720 & 767000 & 3087700 & 3254100 & 3182800 \\
\hline Percentile & 92.8 & 94.1 & 96.5 & 96.7 & 96.6 \\
\hline$\hat{k}_{\text {mean }}$ & -.556 & -.584 & -.525 & -.543 & -.544 \\
\hline$\hat{\sigma}$ & 441970 & 520989 & 3895192 & 4062416 & 3848065 \\
\hline $\begin{array}{l}\hat{\sigma} \text { adjusted to } 99^{\text {th }} \\
\text { percentile }\end{array}$ & 1265689 & 1328770 & 7672348 & 7892341 & 7708540 \\
\hline Share of top 1 percent & 21.4 & 23.6 & 38.3 & 39.5 & 39.0 \\
\hline Gini coefficient & .728 & .739 & .851 & .852 & .858 \\
\hline $\begin{array}{l}\text { Implied population } \\
\text { mean }\end{array}$ & 246451 & 260517 & 677048 & 673568 & 781832 \\
\hline $\begin{array}{l}\text { Number of observations } \\
>\lambda^{0 *}\end{array}$ & 189 & 155 & 1055 & 1030 & 1042 \\
\hline \multicolumn{6}{|c|}{$\begin{array}{l}\text { See notes to table } 1 \text {. The percentiles reported and the adjustment to } \hat{\sigma} \text { are all based on the } \\
\text { observed percentiles. The top } 1 \text { percent is computed allowing for the change in missing } \\
\text { mass. }\end{array}$} \\
\hline
\end{tabular}




\subsection{Estimation, incorporating an external total}

The final approach explored here incorporates the total value of wealth as a constraint on the GPD estimation, using the technique described in section 2.7 above. First, to gain a sense of how well this approach works in reproducing the observed distribution, the unadjusted survey total is used to condition the estimates of the GPD parameters. This approach comes very close to reproducing the estimates from the observed data for the top share, the Gini coefficient and (by construction) mean wealth for both the Austrian data (first column of table 5a) and the SCF (first column of table 5b). The three sets of constrained estimates of $\hat{k}$ and $\hat{\sigma}$ adjusted to $99^{\text {th }}$ percentile are also reasonably similar to those in table 2 for the Austrian data. For the SCF, the three sets of constrained estimates are consistent with each other and with the earlier third set of unconstrained estimates, the only one in that group with any plausibility.

Now, consider the effect of imposing reliable external estimates of the totals, rather than totals estimated from the surveys, while allowing some part of the population at the top of the distribution to be treated as unobserved. The figures taken to represent total household wealth are the ones noted in Section 3.2 in the discussion of the external mean. The same sets of estimates as before are made, assuming a range of unobserved mass at the top of the distribution, from zero for both surveys, to one percent for the Austrian HFCS and 0.1 percent for the SCF. ${ }^{33}$ As with the estimates constrained by the internal survey aggregate estimate, the implied population mean is necessarily identical across all the estimates for each survey.

Perhaps the most dramatic result across the constrained estimates with the Austrian data, regardless of the level of assumed missing mass, is the near doubling of the estimated top share - to a level about 10 percentage points higher than the Vermeulen [2018] estimates from 2010 data from the same survey. In addition, proceeding from zero assumed mass, the data also show a distinct break in the fit at about 0.12 percent assumed missing mass, in terms of how broadly the distribution applies to the data. Below that point, the data used for estimation are very narrowly confined to the top of the observed distribution. At this break, the initial value of $\lambda^{0 *}$ and that of $\lambda^{0 *}$ corresponding to $\hat{k}_{\text {mean }}$ fall considerably, and the sample size available for estimation rises accordingly. For the preferred blended estimates, those for $\lambda^{0 *}$ corresponding to $\hat{k}_{\text {mean }}$, the sample size begins to fall slowly above that level of assumed missing mass. For the estimate for assumed missing mass greater than 0.12 percent, both the Gini coefficient and the top also share decline somewhat for the preferred set of estimates. Using the criterion proposed in the methodology section of this paper, the estimates for 0.12 assumed missing mass are taken as the best estimates by virtue of maximizing the sample support.

In the case of the SCF, the additional fraction of wealth to be accommodated is much smaller than in the Austrian case, though as with Austrian data, imposing the augmented aggregate under any of the missing mass scenarios pushes all of the estimates of $\lambda^{0 *}$ to near or above the estimate for $\lambda^{0 *}$ corresponding to $\hat{k}_{\text {mean }}$ above the top break for the SCF in table 2. ${ }^{34}$ The effect of this compression on the sample size available for estimation is not as limiting as it would be in the Austrian case, however, given the high rate of oversampling in the SCF at the top of the distribution.

Allowing explicitly for the "missing" 400 Forbes people makes virtually no difference in the SCF estimates, relative to no adjustment for missing mass. It may be that even with the high density of very wealthy observations in the SCF sample, the sample is still too small to detect such a small change in the

\footnotetext{
${ }^{33}$ The selection of the highest degree of hypothetical under coverage addressed in the tables is a function of the relative density of observation of the upper tail in the two surveys.

${ }^{34}$ Because all the estimates for $\lambda^{0 *}$ corresponding to $\hat{k}_{\text {mean }}$ and $\lambda^{0 *}$ corresponding to $\hat{k}_{\text {mean }}$ above top break are identical in all cases in table $5 \mathrm{~b}$, the latter estimates are omitted from the table.
} 
assumed coverage. For higher values of assumed missing mass, there are clearer differences. Focusing only on the results for $\lambda^{0 *}$ corresponding to $\hat{k}_{\text {mean }}$, the estimates of $\hat{k}_{\text {mean }}, \hat{\sigma}$, the top share and the Gini coefficient are very stable until around a point where the assumed missing mass is between 0.01 and 0.015 percent. In the points above that, the estimates of $\hat{k}_{\text {mean }}$ (in absolute terms) and the top share decline, as does the supporting sample size. Again, if we accept the criterion of maximizing the sample size to support a choice of assumed missing, the data indicate that a point between 0.01 percent and 0.015 percent missing mass would be preferred.

To give a sense of the precision of the estimates with an imposed implied aggregate, we take 0.2 percent for the Austrian HFCS and 0.01 percent for the SCF as working estimates of the extent of effective under coverage. Appendix table A1 provides key estimates pooled over implicates and the associated standard errors with respect to imputation and sampling. For the Austrian HFCS, the estimated standard errors associated with the top share are substantially reduced relative to either the full-sample estimate or the unadjusted model reported in table 2. The standard error for the Gini coefficient is only marginally different in these cases, though the estimated values are larger for this final model. The mean, being constrained by the imposition of an aggregate amount, has no variation. In the case of the SCF, the standard errors and the mean estimates for the top share and the Gini coefficient are very close to those from the unadjusted data; ${ }^{35}$ this might be expected, given the relatively small adjustment made to the implied SCF aggregate. Focusing on the estimates for $\lambda^{0 *}$ corresponding to $\hat{k}_{\text {mean }}$ above top break, those estimates do not differ greatly from those for the unadjusted model, but the standard errors are disproportionately smaller. Overall, there is a precision gain from imposing an external aggregate, as might be expected.

\footnotetext{
${ }^{35}$ Note that because the aggregate imposed on the SCF estimation here was based on a percentage addition to the sample estimate, the mean has some variation across both the implicates and the sample replicates.
} 
Table 5a: Parameter estimates and related statistics, for various values of $\lambda^{0 *}$, Austrian HFCS, where estimation is conditioned on a specific value of wealth and various fractions of the population are treated as unobserved.

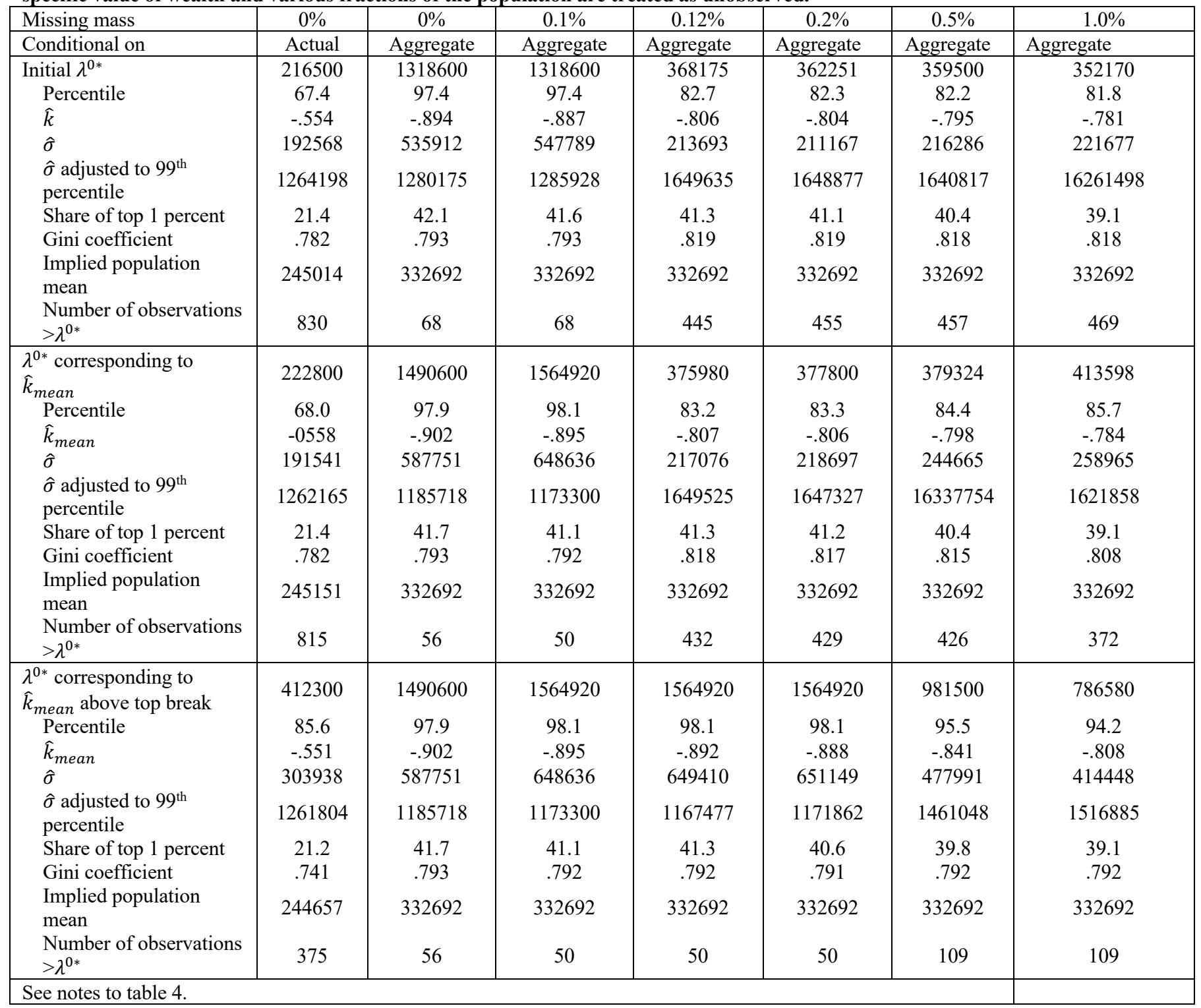


Table 5b: Parameter estimates and related statistics, for various values of $\lambda^{*}$, SCF, where estimation is conditioned on a specific value of wealth and various fractions of the population are treated as unobserved.

\begin{tabular}{|c|c|c|c|c|c|c|c|}
\hline Missing mass & $0 \%$ & $0 \%$ & $0.00032 \%$ & $0.01 \%$ & $0.015 \%$ & $0.05 \%$ & $0.1 \%$ \\
\hline Conditional on & Actual & Aggregate & Aggregate & Aggregate & Aggregate & Aggregate & Aggregate \\
\hline Percentile & 96.4 & 96.4 & 96.4 & 96.4 & 96.4 & 96.6 & 96.6 \\
\hline$\hat{\sigma}$ & 3826086 & 3823841 & 3824332 & 3830930 & 3833550 & 4033332 & 4061544 \\
\hline$\hat{\sigma}$ adjusted to $99^{\text {th }}$ percentile & 7730137 & 7733153 & 7732824 & 7721881 & 7716120 & 7685078 & 7730137 \\
\hline Share of top 1 percent & 38.7 & 38.8 & 38.8 & 38.7 & 38.6 & 38.3 & 37.8 \\
\hline $\begin{array}{l}\text { Number of observations } \\
>\lambda^{*}\end{array}$ & 1067 & 1067 & 1067 & 1067 & 1067 & 1042 & 1042 \\
\hline$\lambda^{*}$ corresponding to $\hat{k}_{\text {mean }}$ & 2980960 & 2980960 & 2980960 & 3087700 & 4694900 & 3832200 & 5062500 \\
\hline Percentile & 96.4 & 96.4 & 96.4 & 96.5 & 96.6 & 97.2 & 97.8 \\
\hline$\hat{k}_{\text {mean }}$ & -.535 & -.535 & -.535 & -.532 & -0.533 & -0.505 & -.485 \\
\hline$\hat{\sigma}$ & 3826086 & 3823841 & 3824332 & 399006 & 4774503 & 4647469 & 5230427 \\
\hline $\begin{array}{l}\text { Number of observations } \\
>\lambda^{*}\end{array}$ & 1067 & 1067 & 1067 & 1055 & 908 & 964 & 880 \\
\hline $\begin{array}{l}\lambda^{*} \text { corresponding to } \\
\hat{k}_{\text {mean }} \text { above top break }\end{array}$ & 2980960 & 2980960 & 2980960 & 3087700 & 4694900 & 3832200 & 5062500 \\
\hline Percentile & 96.4 & 96.4 & 96.4 & 96.5 & 96.6 & 97.2 & 97.8 \\
\hline$\hat{k}_{\text {mean }}$ & -.535 & -.535 & -.535 & -.532 & -0.533 & -0.505 & -.485 \\
\hline$\hat{\sigma}$ & 3826086 & 3823841 & 3824332 & 399006 & 4774503 & 4647469 & 5230427 \\
\hline$\hat{\sigma}$ adjusted to $99^{\text {th }}$ percentile & 7730137 & 7733153 & 7732824 & 7728414 & 7750610 & 7940401 & 7763226 \\
\hline Share of top 1 percent & 38.7 & 38.8 & 38.8 & 38.7 & 38.8 & 38.5 & 37.7 \\
\hline Gini coefficient & .852 & .852 & .852 & .852 & 0.851 & 0.851 & .850 \\
\hline Implied population mean & 679847 & 680132 & 680132 & 680132 & 680132 & 680132 & 680132 \\
\hline
\end{tabular}

\section{Summary and conclusions}

Many surveys now collect data on wealth, and those data are often used to characterize inequality. There is also growing interest in using such information in the construction of distributional national accounts. However, owing in varying degrees to thinness of sample coverage of the upper tail of the wealth distribution and differential nonresponse among that group, survey measurements of inequality may often be biased, or otherwise incomparable, and survey-based estimates of means may fall noticeably short of comparable aggregate figures (Kennickell, 2019). Fitting a parametric distribution to the upper tail is one tool that may be used to address these problems. Most such work has involved use of the simple Pareto distribution, sometimes employing information from "rich lists" to supplement the observed data. Unfortunately, the construction of such lists is generally opaque, and some research indicates they may seriously misrepresent the upper tail.

This paper takes a different path, using the generalized Pareto distribution (GPD), a more flexible functional form that encompasses the simple Pareto. This method, as implemented here, allows for coping explicitly with potential mismeasurement of the extreme upper tail of the distribution through measurement error or effective under coverage. Importantly, it also provides a way to incorporate knowledge of the aggregate amount of wealth as a constraint on the estimation, rather than relying on 
questionable data from rich lists; this feature seems likely to have the greatest practical application, particularly in the construction of distributional national accounts.

The 2017 Austrian implementation of the Household Finance and Consumption Survey and the 2016 U.S. Survey of Consumer Finances serve here as the empirical basis for exploring the effectiveness of this approach. Although both surveys are specifically designed to measure household wealth and they have other important methodological characteristics in common, they differ importantly in terms of their sample designs: as a consequence of its use of administrative data in sampling and nonresponse adjustment, the SCF is able to provide a much denser representation of the extreme upper tail of the wealth distribution. As a possible consequence, the SCF also yields an estimate of total household wealth much closer to the independently estimated aggregate figure than is the case for the Austrian HFCS. The SCF, however, explicitly excludes the very wealthiest U.S families, as determined according to a "rich list", and there is some indication that it may deviate from the expected distribution at the extreme top of the distribution, even when those households are excluded (see Kennickell, 2017).

Ideally, fitting a GPD to the survey data would result in increasing the alignment of implied survey wealth aggregates with external aggregates. The results of straightforward fitting of the wealth data using a GPD without adjustments suggests that the GPD can provide a good description of the data; the model implies that about the top 20 percent of households can be modeled with the distribution. However, closer examination reveals problems. Although the model closely reproduces key distributional characteristics (mean, top share, Gini coefficient) of the Austrian wealth distribution measured directly from the survey data, it makes a negligible difference in addressing the understatement of aggregate wealth as measured by the survey, relative to an approximately comparable aggregate value. In the case of the SCF, the most straightforward GPD estimates imply an implausibly greater degree of wealth concentration than that implied by direct estimation, and an aggregate far beyond the national accounting measure; restricting the support of estimation to the upper reaches of the observed distribution yields results that, as in the case of the Austrian data, closely reproduce the estimates made directly from the data, while falling (less) short of the aggregate wealth measure.

We are then able to ask: is the failure of the straightforward estimation to close the gap with the aggregate a function of measurement problems of some sort in the observed upper tail? Re-estimating the models while excluding various extents of the extreme of the observed upper tail of the wealth distribution makes some difference, but not of a sort that provides a great or consistent improvement relative to the overall picture from the more straightforward estimation. In the case of the Austrian data, excluding larger amounts of data from the estimation does imply a higher degree of wealth concentration and a higher value of the mean value, but the change is not enough to bridge the gap to the value of the external aggregate. In the case of the SCF, excluding the wealthiest one-half percent amplifies the implausibility of the most straightforward estimates and decreases implied concentration for the estimates more focused on the top of the distribution; excluding the wealthiest one percent further increases the implausible estimates and increases the concentration measures for the estimates more focused on the top. While there may well be important measurements problems with the largest wealth values as measured in the surveys, more detailed investigation is needed to understand the nature of such problems and the extent to which omitting observations may be helpful for GPD estimation.

Alternatively, it may be that a meaningful part of the upper tail is not captured in the surveys at all. The approach developed in this paper allows for a relatively straightforward means of allowing for effective under coverage, by effectively uniformly compressing the observed distribution into a smaller range and treating the remaining range at the top explicitly as unobserved. For both surveys considered, allowing for an unobserved population at the top can make a substantial difference in the estimates and yield a 
closer match to the aggregates. Unfortunately, the degree of difference does not appear to be sufficiently stable or consistent for one to expect this method to be usable alone to align the observed data in other surveys with external aggregates. Further work with this model should explore the joint possibility of mismeasurement in the observations of the upper tail and effective under coverage of that population.

The final approach considered is to take an external measure of aggregate of wealth as a constraint in estimation and consider a range of fractions of the upper tail that might have been unobserved. It is clear from the results that it is possible to fit a GPD for each set of wealth data in a way that incorporates more than a small number of observations at the very top of the observed distribution. In the case of the Austrian data, assuming that only 0.12 percent of the population at the top of the wealth distribution is unobserved allows the GPD to be fit over the top 17 percent of the observed wealth distribution.

Regardless of the extent of the under coverage assumed, the wealth share of the top one percent nearly doubles, relative to the figure estimated directly from the survey-actually exceeding the SCF fraction in terms of point estimates. The changes for the SCF are less dramatic, again largely because the extent to which the unadjusted wealth data already line up with the comparable aggregate is so much more closely. Given the high degree of over sampling of the wealthy in that survey, it is not a surprise that the estimates indicate that only perhaps around 0.01 percent of the tail may be unobserved, a small but implicitly very wealthy group. For countries with reliable aggregate wealth data or other means of constructing a sufficiently reliable proxy, this approach may be preferable to relying on "rich list" data, which are most often assembled in a way far less transparent than in the case of national accounting data.

This work was motivated in large part by a desire to provide a convenient and principled means of making more comparable inequality estimates from as many of the component surveys of the HFCS as possible. Effort toward this end will be a next step for this work. The approach may also be helpful in countries where there are already fairly well aligned survey and aggregate wealth measures for at least one survey, for example, as in the case of the U.S. Pfeffer et al. [2016] discuss the difference between wealth distributions in the SCF and the Panel Study on Income Dynamics (PSID) and the role played by oversampling in the SCF. Using the methods developed here to "tune" the GPD model to reproduce the SCF results with the PSID might allow a reweighting of the PSID in a way to better characterize the population it describes.

A GPD, or any other extreme-value distribution, fitted to empirical wealth data is only an approximation. Generally, such distributions imply that there is nonzero mass at arbitrarily large values. In the examples developed in this paper, the number of households implied to be beyond $10^{8}$, a not at all implausible figure, is very much less than one and the corresponding fraction of wealth is not large enough to disrupt the qualitative results. In other applications, it would be important to be sure that this also hold.

The applications presented here have assumed that the possibility of substantial under-reporting of values obtained in the surveys can safely be ignored. In very highly skewed distributions, such as that for wealth, the largest values tend to have a strongly disproportionate effect. Nonetheless, in some surveys underreporting of smaller values may still account for a substantial fraction of any deviation between an aggregate based solely on survey data and one from a national account. A GPD tail fitted to such data would be biased, especially if the estimation method developed here to incorporate an external aggregate were used. An appropriate model to account for underreporting should be developed and applied to the data before attempting to incorporate a fitted tail of any sort.

Finally, for many purposes it is not sufficient to use the GPD method here just to generate a distribution of total wealth that aligns with national account data: components of wealth (and other characteristics) are also important, and their totals may differ from their own national account counterpart. But constructing 
distributions of wealth components that also align with aggregates is complicated by the fact that not everyone necessarily holds each component, and the positive portfolio shares may well vary with variables other than total wealth. While some of the techniques presented in this paper might be helpful in addressing this problem, it may be best to consider first the specific estimation needs and whether approaches other than creating a more broadly synthetic upper tail might serve those needs. 


\section{Bibliography}

Albacete, Nicolás, Sarah Thandi Dippenaar, Peter Lindner, Karin Wagner [2019] "Eurosystem Household Finance and Consumption Survey 2017: Methodological Notes for Austria", Oesterreichische National Bank.

Albacete, N., P. Lindner, K. Wagner, and S. Zottel, "Household Finance and Consumption Survey des Eurosystems 2010: Methodische Grundlagen für Österreich,” Geldpolitik \& Wirtschaft, 3/2012 Addendum, 2012.

Antoniewicz, R. 2000. "A Comparison of the Household Sector from the Flow of Funds Accounts and the Survey of Consumer Finances." Federal Reserve Board of Governors, Survey of Consumer Finances Working Paper. Available at: https://www.federalreserve.gov/econresdata/scf/files/antoniewicz_paper.pdf.

Atkinson, A. B. (1975). "The distribution of wealth in Britain in the 1960s the estate duty method reexamined". In The Personal Distribution of Income and Wealth, NBER, James D. Smith (ed.), pp. 277328.

Avery, Robert B, Gregory E. Elliehausen and Arthur B. Kennickell [1988] "Measuring Wealth with Survey Data: An Evaluation of the 1983 Survey of Consumer Finances," Review of Income and Wealth, (December), pp. 339-369.

Batty, Michael M., Jesse Bricker, Joseph S. Briggs, Elizabeth Holmquist, Susan Hume McIntosh, Kevin B. Moore, Eric R. Nielsen, Sarah Reber, Molly Shatto, Kamila Sommer, Tom Sweeney, and Alice Henriques Volz [2019] "Introducing the Distributional Financial Accounts of the United States" Federal Reserve Board Finance and Economic Discussion Series 2019-017, https://doi.org/10.17016/FEDS.2019.017.

Blanchet, Thomas, Bertrand Garbinti, Jonathan Goupille-Lebret, and Clara Martínez-Toledano [2018] "Applying Generalized Pareto Curves to Inequality Analysis." AEA Papers and Proceedings, 108: 11418. DOI: $10.1257 /$ pandp. 20181075

Bricker, Hansen and Volz [2019] "Wealth concentration in the U.S. after augmenting the upper tail of the Survey of Consumer Finances”, Economics Letters 184, https://doi.org/10.1016/j.econlet.2019.108659.

Brazauskas, Vytaras and Andreas Kleefeld [2009] "Robust and Efficient Fitting of the Generalized Pareto Distribution with Actuarial Applications in View", Insurance: Mathematics and Economics, Volume 45, Issue 3, December 2009, Pages 424-435. https://doi.org/10.1016/j.insmatheco.2009.09.002

Cantarella, Michele, Andrea Neri and Maria Giovanna Ranalli [2021] "Mind the wealth gap: a new allocation method to match micro and macro statistics for household wealth" working paper, arXiv:2101.01085v1.

Enrique Castillo \& Ali S. Hadi (1997) Fitting the Generalized Pareto Distribution to Data, Journal of the American Statistical Association, 92:440, 1609-1620, DOI: 10.1080/01621459.1997.10473683.

Chakraborty, R. and Waltl, S. R. (2018). "Missing the wealthy in the HFCS: micro problems with macro implications". ECB Working Paper Series No 2163, European Central Bank. 
Anna Chernobai, Anna, Svetlozar Rachev, and Frank Fabozzi (2005) "Composite Goodness-of-Fit Tests for Left-Truncated Loss Samples”, mimeo, Department of Statistics and Applied Probability, University of California, Santa Barbara.

V Choulakian \& M. A Stephens (2001) Goodness-of-Fit Tests for the Generalized Pareto Distribution, Technometrics, 43:4, 478-484, DOI: 10.1198/00401700152672573.

Dettling, L.J., S.J. Devlin-Foltz, J. Krimmel, S.J. Pack, and J.P. Thompson. 2015. “Comparing Micro and Macro Sources for Household Accounts in the United States: Evidence from the Survey of Consumer Finances." Finance and Economics Discussion Series 2015-086. Washington: Board of Governors of the Federal Reserve System, Doi: http://dx.doi.org/10.17016/FEDS.2015.086.

European Central Bank [2020a] "The Household Finance and Consumption Survey: Methodological report for the 2017 wave" Statistical Paper Series, No. 35 (March):

https://www.ecb.europa.eu/pub/pdf/scpsps/ecb.sps35 b9b07dc66d.en.pdf?8fcb3cd59213bac0784168618a $\underline{9 \mathrm{~b} 5 \mathrm{fb} 3}$.

European Central Bank [2020b] "The Household Finance and Consumption Survey: Results from the 2017 wave" Statistical Paper Series, No. 36 (March):

https://www.ecb.europa.eu/pub/pdf/scpsps/ecb.sps36 0245ed80c7.en.pdf?bd73411fbeb0a33928ce4c5ef2 c5e872.

Fessler, Pirmin, Peter Lindner, Martin Schürz [2019] "Eurosystem Household Finance and Consumption Survey 2017 for Austria", Oesterreichische National Bank, https:/www.oenb.at/dam/jcr:8b5436a372a2-415a-a0b3-ea476c371a0c/4_mop_\%20q4_18_screen.pdf.

Henriques, Alice M. and Joanne W. Hsu [2014] "Analysis of Wealth using Micro and Macro Data: A Comparison of the Survey of Consumer Finances and Flow of Funds Accounts," in Jorgenson, Dale W., J.S. Landefeld and Paul Schreyer, eds., Measuring Economic Sustainability and Progress, Studies in Income and Wealth, Volume 72, Cambridge, MA: National Bureau of Economic Research.

Hoffmann, I., Börner, C.J. (2020) The risk function of the goodness-of-fit tests for tail models. Stat Papers (2020). https://doi.org/10.1007/s00362-020-01159-3.

Kavonius, I.K. and V.M. Törmälehto. 2010. "Integrating Micro and Macro Accounts - The Linkages between Euro Area Household Wealth Survey and Aggregate Balance Sheets for Households." IARIW 31st General Conference, St Gallen, Switzerland, August 22-28, 2010, http://www.iariw.org/papers/2010/7akavonius.pdf.

Kennickell, A.B. The tail that wags: differences in effective right tail coverage and estimates of wealth inequality. J Econ Inequal 17, 443-459 (2019). https://doi.org/10.1007/s10888-019-09424-8

Kennickell, A.B. [2017] "Lining up: Survey and administrative data estimates of wealth concentration”, Statistical Journal of the IAOS, vol. 33, no. 1, pp. 59-79, 2017. https://doi.org/10.3233/SJI-170349

Kennickell, A.B. [1999] "Using Income Data to Predict Wealth," working paper, Board of Governors of the Federal Reserve System, https://www.federalreserve.gov/econresdata/scf/files/wealthincome7web.pdf.

Kish, Leslie (1965). Survey Sampling. New York: Wiley. 
Langousis, A., A. Mamalakis, M. Puliga, and R. Deidda (2016), Threshold detection for the generalized Pareto distribution: Review of representative methods and application to the NOAA NCDC daily rainfall database, Water Resourses Research, 52, 2659-2681, doi:10.1002/2015WR018502.

Oesterreichische National Bank [2019] Household income, consumption and wealth: Austrian sector accounts 1998-2017, (April), www.oenb.at/en/Publications/Statistics/Special-Issues/2019/sectoraccounts-2019.html.

Pfeffer, Fabian T., Robert F. Schoeni Arthur B. Kennickell and Patricia Andreski [2016] "Measuring wealth and wealth inequality: Comparing two U.S. surveys," Journal of Economic and Social Measurement, 41, 2016, pp. 103-120.

Pickands, James. [1965] "Statistical Inference Using Extreme Order Statistics.” Annals of Statistics 3, no. 1, 119—131. doi:10.1214/aos/1176343003. https://projecteuclid.org/euclid.aos/1176343003

Pham, M. H., Tsokos, C., \& Choi, B.-J. (2018). Maximum likelihood estimation for the generalized Pareto distribution and goodness-of-fit test with censored data. Journal of Modern Applied Statistical Methods, 17(2), eP2608. doi: 10.22237/jmasm/1553261471

Raub, Brian, Barry Johnson, and Joseph Newcomb [2010] "A Comparison of Wealth Estimates for America's Wealthiest Decedents, Using Tax Data and Data from the Forbes 400", Proceedings of the $103^{\text {rd }}$ Annual Conference on Taxation (Chicago), 2010, National Tax Association, https://ntanet.org/wpcontent/uploads/proceedings/2010/020-raub-a-comparison-wealth-2010-nta-proceedings.pdf.

Vermeulen, P. (2018). "How fat is the top tail of the wealth distribution?" Review of Income and Wealth, 64(2):357-387. 


\section{Appendix A: Selected standard errors with respect to imputation and sampling}

Table A1: Selected estimates and confidence intervals, based on survey implicates and sample replicates, Austrian HFCS and SCF.

\begin{tabular}{|c|c|c|c|c|}
\hline & No adjustments & $\begin{array}{l}\text { Imposed aggregate, } \\
0.2 \% \text { missing mass }\end{array}$ & No adjustments & $\begin{array}{l}\text { Measured amt. }+4.2 \% \\
0.01 \% \text { missing mass }\end{array}$ \\
\hline & \multicolumn{2}{|c|}{ Austrian HFCS } & \multicolumn{2}{|r|}{ SCF } \\
\hline \multicolumn{5}{|c|}{ First acceptable $\lambda^{*}$} \\
\hline Percentile & $\begin{array}{l}66.9 \\
2.00\end{array}$ & $\begin{array}{r}93.8 \\
9.56\end{array}$ & $\begin{array}{l}72.4 \\
5.74\end{array}$ & $\begin{array}{l}96.4 \\
3.06\end{array}$ \\
\hline$\widehat{k}$ & $\begin{array}{c}-0.522 \\
0.053\end{array}$ & $\begin{array}{c}-0.872 \\
0.052\end{array}$ & $\begin{array}{c}-0.822 \\
0.033\end{array}$ & $\begin{array}{c}-0.539 \\
0.048\end{array}$ \\
\hline$\hat{\sigma}$ adjusted to $99^{\text {th }}$ percentile & $\begin{array}{c}1194783 \\
134237\end{array}$ & $\begin{array}{c}1345812 \\
234741\end{array}$ & $\begin{array}{l}8827098 \\
430802\end{array}$ & $\begin{array}{c}7791434 \\
523866\end{array}$ \\
\hline Share of top 1 percent & $\begin{array}{l}19.7 \\
2.79\end{array}$ & $\begin{array}{r}41.3 \\
1.94\end{array}$ & $\begin{array}{l}57.7 \\
5.47\end{array}$ & $\begin{array}{l}38.7 \\
0.617\end{array}$ \\
\hline Gini coefficient & $\begin{array}{r}0.776 \\
0.011\end{array}$ & $\begin{array}{r}0.798 \\
0.018\end{array}$ & $\begin{array}{r}0.915 \\
0.012\end{array}$ & $\begin{array}{c}0.854 \\
0.005\end{array}$ \\
\hline Implied population mean & $\begin{array}{c}239266 \\
12735 \\
\end{array}$ & $\begin{array}{c}332692 \\
0 \\
\end{array}$ & $\begin{array}{r}979269 \\
153263 \\
\end{array}$ & $\begin{array}{c}689798 \\
14099 \\
\end{array}$ \\
\hline \multicolumn{5}{|c|}{$\lambda^{*}$ corresponding to $\hat{k}_{\text {mean }}$} \\
\hline Percentile & $\begin{array}{l}80.5 \\
10.3\end{array}$ & $\begin{array}{l}94.4 \\
8.71\end{array}$ & $\begin{array}{l}81.0 \\
4.88\end{array}$ & $\begin{array}{l}97.4 \\
4.40\end{array}$ \\
\hline$\widehat{k}$ & $\begin{array}{l}-0.55 \\
0.077\end{array}$ & $\begin{array}{c}-0.876 \\
0.052\end{array}$ & $\begin{array}{c}-0.785 \\
0.036\end{array}$ & $\begin{array}{c}-0.534 \\
0.052\end{array}$ \\
\hline$\hat{\sigma}$ adjusted to $99^{\text {th }}$ percentile & $\begin{array}{c}1222412 \\
231077\end{array}$ & $\begin{array}{c}1312376 \\
239397\end{array}$ & $\begin{array}{c}8517548 \\
406712\end{array}$ & $\begin{array}{c}7784971 \\
504317\end{array}$ \\
\hline Share of top 1 percent & $\begin{array}{r}20.7 \\
3.38\end{array}$ & $\begin{array}{r}41.2 \\
1.88\end{array}$ & $\begin{array}{r}52.7 \\
5.00\end{array}$ & $\begin{array}{l}38.7 \\
0.703\end{array}$ \\
\hline Gini coefficient & $\begin{array}{r}0.749 \\
0.022\end{array}$ & $\begin{array}{r}0.797 \\
0.016\end{array}$ & $\begin{array}{r}0.900 \\
0.012\end{array}$ & $\begin{array}{r}0.853 \\
0.005\end{array}$ \\
\hline Implied population mean & $\begin{array}{c}242609 \\
13832 \\
\end{array}$ & $\begin{array}{c}332692 \\
0 \\
\end{array}$ & $\begin{array}{r}882118 \\
109651 \\
\end{array}$ & $\begin{array}{c}689798 \\
14099 \\
\end{array}$ \\
\hline \multicolumn{5}{|c|}{$\lambda^{*}$ corresponding to $\hat{k}_{\text {mean }}$ above top break } \\
\hline Percentile & $\begin{array}{l}92.4 \\
2.76\end{array}$ & $\begin{array}{r}97.4 \\
4.01\end{array}$ & $\begin{array}{r}97.5 \\
1.54\end{array}$ & $\begin{array}{l}97.4 \\
1.40\end{array}$ \\
\hline 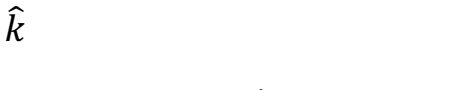 & $\begin{array}{c}-0.516 \\
0.084\end{array}$ & $\begin{array}{c}-0.892 \\
0.029\end{array}$ & $\begin{array}{c}-0.536 \\
0.065\end{array}$ & $\begin{array}{c}-0.534 \\
0.033\end{array}$ \\
\hline$\hat{\sigma}$ adjusted to $99^{\text {th }}$ percentile & $\begin{array}{c}1196111 \\
139232\end{array}$ & $\begin{array}{c}1317268 \\
186922\end{array}$ & $\begin{array}{c}7708380 \\
424001\end{array}$ & $\begin{array}{c}7784971 \\
389327\end{array}$ \\
\hline Share of top 1 percent & $\begin{array}{l}19.8 \\
3.27\end{array}$ & $\begin{array}{r}41.1 \\
1.94\end{array}$ & $\begin{array}{r}38.5 \\
1.51\end{array}$ & $\begin{array}{l}38.7 \\
0.692\end{array}$ \\
\hline Gini coefficient & $\begin{array}{r}0.721 \\
0.016\end{array}$ & $\begin{array}{r}0.792 \\
0.009\end{array}$ & $\begin{array}{l}0.853 \\
0.004\end{array}$ & $\begin{array}{r}0.853 \\
0.003\end{array}$ \\
\hline Implied population mean & $\begin{array}{c}239602 \\
11110 \\
\end{array}$ & $\begin{array}{c}332692 \\
0\end{array}$ & $\begin{array}{c}687132 \\
22747\end{array}$ & $\begin{array}{c}689798 \\
14099 \\
\end{array}$ \\
\hline
\end{tabular}




\section{Appendix B: Distributional Summary Statistics}

Using survey data together with GPD estimates of the upper tail of a distribution to compute summary distributional measures typically used to quantify aspects of inequality - such as concentration ratios, quantile ratios and Gini coefficients - requires a way of blending the two sources. This appendix outlines the approach to estimate the measures reported in this paper.

\section{Quantile ratios of the full distribution}

Using the notation in the body of this paper, let $\Pi(\lambda)$ represent the percentile corresponding to $\lambda$ in the complete distribution of $\lambda$, not just the part fit using the GPD. If $\Pi(\lambda) \leq \Pi\left(\lambda^{*}\right)$, then the value of $\lambda$ is directly observable. If $\Pi(\lambda)>\Pi\left(\lambda^{*}\right)$, the value of $\lambda$ is computed using the inverted version of $(1)$ and estimated GPD parameters as follows:

$$
\lambda(\Pi)=\frac{\sigma}{k}\left\{1-\left[1-\frac{\Pi-\Pi\left(\lambda^{*}\right)}{100-\Pi\left(\lambda^{*}\right)}\right]^{k}\right\}+\lambda^{*}, \text { for } \Pi>\Pi\left(\lambda^{*}\right) .
$$

The quantile ratios, for example the P99/P25 ratio, are defined using the actual or simulated values, as appropriate.

\section{Concentration ratios}

Let the total population be given by $\Omega$. Then the overall total amount of $\mathrm{X}$ is given by

$$
T=\sum_{\lambda<\lambda^{*}} W(\lambda) \lambda+\Omega\left(1-\frac{\Pi\left(\lambda^{*}\right)}{100}\right)\left(\frac{\sigma}{1+k}+\lambda^{*}\right)
$$

If $\Pi_{1}<\Pi_{2} \leq \Pi\left(\lambda^{*}\right)$, the sum of $X$ held by the group in the percentile range $\left(\Pi_{1}, \Pi_{2}\right)$ may be computed directly from the observed data and the share determined by dividing by $\mathrm{T}$. If $\Pi_{4},>\Pi_{3} \geq \Pi\left(\lambda^{*}\right)$, the definition of the GPD mean given in (8) can be used to derive the share for the percentile range $\left(\Pi_{3}, \Pi_{4}\right)$ as follows:

$\mathrm{S}\left(\Pi_{3}, \Pi_{4}\right)=\Omega\left\{\left(1-\frac{\Pi_{3}}{100}\right)\left[\frac{\sigma-k\left(\lambda\left(\Pi_{3}\right)-\lambda^{*}\right)}{1+k}+\mathrm{X}\left(\Pi_{3}\right)\right]-\left(1-\frac{\Pi_{4}}{100}\right)\left[\frac{\sigma-k\left(\lambda\left(\Pi_{4}\right)-\lambda^{*}\right)}{1+k}+\lambda\left(\Pi_{4}\right)\right]\right\} / T$.

If the interval $\left(\Pi_{3}, \Pi_{4}\right)$ contains $\Pi\left(\lambda^{*}\right)$, then the first half of difference given inside the curly braces in (B3) is replaced by

$$
\left(\frac{\Pi\left(\lambda^{*}\right)-\Pi_{3}}{100}\right) \sum_{\lambda=\lambda\left(\Pi_{3}\right)}^{\lambda^{*}} W(\lambda) \lambda+\left(1-\frac{\Pi\left(\lambda^{*}\right)}{100}\right)\left[\frac{\sigma}{1+k}+\lambda^{*}\right]
$$

\section{Gini coefficient}

The Gini coefficient may be computed as one minus twice the area under the Lorenz curve, the cumulative share of $\lambda$ plotted against the corresponding cumulative share of the population. Figure B1 illustrates how the calculation of the area can be partitioned into three parts that can be computed using the observed data and the estimated GPD parameters. 


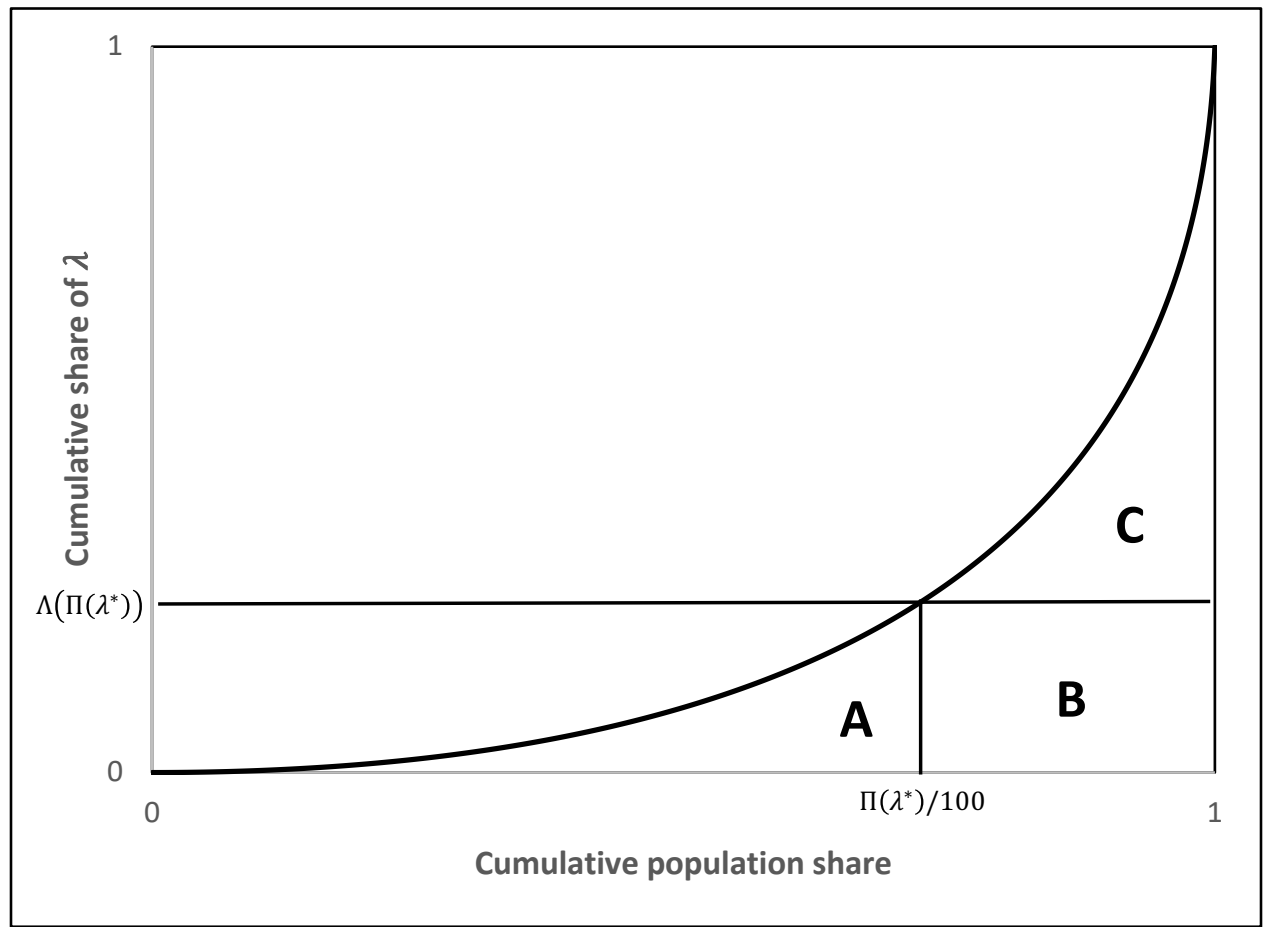

Area $\mathrm{A}$ in the figure covers the cumulative population range $\left(0, \Pi\left(\lambda^{*}\right) / 100\right)$ and the cumulative share of $\lambda$ in the range $\left(0, \Lambda\left(\Pi\left(\lambda^{*}\right)\right)\right)$, where $\Lambda$ is the mapping from a given percentile to the corresponding cumulative share of the value of $\lambda$. The shares of $\lambda$ are defined relative to $T$ given in (B2). This area can be computed directly from the observed data and the value of $\mathrm{T}$. The rectangular area $\mathrm{B}$ is the product $\left(1-\Pi\left(\lambda^{*}\right) / 100\right) \Lambda\left(\Pi\left(\lambda^{*}\right)\right)$. Area $\mathrm{C}$ is area under a Lorenz curve defined by the estimated GPD, rescaled by $\left(1-\Pi\left(\lambda^{*}\right) / 100\right)\left(1-\Lambda\left(\Pi\left(\lambda^{*}\right)\right)\right)$ to account for its relative contribution to the estimate of the area under the overall Lorenz curve. This quantity is computed as follows:

$$
\begin{gathered}
C=\left[1-\Pi\left(\lambda^{*}\right) / 100\right]\left[1-\Lambda\left(\Pi\left(\lambda^{*}\right)\right)\right] \int_{0}^{1} \int_{0}^{\propto} \frac{\sigma}{\frac{k}{k}\left[1-(1-\mathrm{p})^{k}\right]} \\
\sigma /(1+k) \\
=\left[1-\Pi\left(\lambda^{*}\right) / 100\right]\left[1-\Lambda\left(\Pi\left(\lambda^{*}\right)\right)\right] \frac{k+1}{2 k+4}, k>-1
\end{gathered}
$$

The overall Gini coefficient is given by $1-2(A+B+C)$. 